\title{
A Semi-Exact Algorithm for Quickly Computing a Maximum Weight Clique in Large Sparse Graphs
}

\author{
Shaowei Cai \\ Jinkun Lin \\ State Key Laboratory of Computer Science \\ Institute of Software \\ Chinese Academy of Sciences \\ Beijing, China
}

Yiyuan Wang

School of Computer Science and Information Technology

Northeast Normal University, China
SHAOWEICAI.CS@GMAIL.COM

JKUNLIN@GMAIL.COM

YIYUANWANGJLU@126.COM

DSTRASH@HAMILTON.EDU

Department of Computer Science

Hamilton College, Clinton, NY, USA

\begin{abstract}
This paper explores techniques to quickly solve the maximum weight clique problem (MWCP) in very large scale sparse graphs. Due to their size, and the hardness of MWCP, it is infeasible to solve many of these graphs with exact algorithms. Although recent heuristic algorithms make progress in solving MWCP in large graphs, they still need considerable time to get a high-quality solution. In this work, we focus on solving MWCP for large sparse graphs within a short time limit. We propose a new method for MWCP which interleaves clique finding with data reduction rules. We propose novel ideas to make this process efficient, and develop an algorithm called FastWClq. Experiments on a broad range of large sparse graphs show that FastWClq finds better solutions than state-of-the-art algorithms while the running time of FastWClq is much shorter than the competitors for most instances. Further, FastWClq proves the optimality of its solutions for roughly half of the graphs, all with at least $10^{5}$ vertices, with an average time of 21 seconds.
\end{abstract}

\section{Introduction}

The proliferation of large data sets brings with it a series of special computational challenges. Many data sets can be modeled as graphs, and the research of large real-world graphs has grown enormously in last decade. A clique of a graph is a subset of the vertices that are all pairwise adjacent. Cliques are an important graph-theoretic concept, and are often used to represent dense clusters. The maximum clique problem (MCP) is a long-standing problem in graph theory, for which the task is to find a clique with the maximum number of vertices in the given graph. An important generalization of MCP is the maximum weight clique problem (MWCP), in which each vertex is associated with a positive integer weight, and the goal is to find a clique with the largest weight. MWCP has valuable applications in many fields (Ballard \& Brown, 1982; Balasundaram \& Butenko, 2006; Gomez Ravetti \& Moscato, 2008). 
The decision version of MCP (and thus MWCP) is one of Karp's prominent 21 NP-complete problems (Karp, 1972), and is complete for the class W[1], the parameterized analog of NP (Fellows $\&$ Downey, 1998). Moreover, MCP (and thus MWCP) is not approximable within $n^{1-\varepsilon}$ for any $\varepsilon>0$ unless $\mathrm{P}=\mathrm{NP}$ (Zuckerman, 2007). Nevertheless, these negative theoretical results have been established for a "worst case" that is not often observed in practice. We therefore still have hope of solving MWCP problems which arise in specific problem domains.

\subsection{Related Work}

Given their theoretical importance and practical relevance, considerable effort has been devoted to the development of various methods for MCP and MWCP, mainly including exact algorithms and heuristic algorithms. Exact algorithms are dedicated to find an exact solution and prove its optimality, while heuristic algorithms have been devised with the purpose of providing (potentially) sub-optimal solutions within an acceptable time.

Almost all existing exact algorithms for $\mathrm{MCP}$ are branch-and-bound $(\mathrm{BnB})$ algorithms, and they differ from each other mainly by their techniques to determine the upper bounds and their branching strategies. A large family of $\mathrm{BnB}$ algorithms use coloring to compute upper bounds (Tomita \& Seki, 2003; Tomita \& Kameda, 2007; Konc \& Janezic, 2007; Tomita et al., 2010; San Segundo et al., 2013). Another paradigm encodes MCP into a MaxSAT instance and then applies MaxSAT reasoning to improve the upper bound (Li \& Quan, 2010; Li, Fang, \& Xu, 2013; Li, Jiang, \& Manyà, 2017).

There are also numerous works on heuristic algorithms for MCP, most of which are local search algorithms (Singh \& Gupta, 2006b; Pullan \& Hoos, 2006; Pullan, 2006; Guturu \& Dantu, 2008; Benlic \& Hao, 2013). A milestone local search algorithm for MCP is the Dynamic Local Search (DLS) algorithm due to Pullan and Hoos (2006), which was later improved to the Phased Local Search (PLS) algorithm by Pullan (2006). Pullan (2009) later adapted PLS to weighted problems.

Recently, there have been some dedicated algorithms for solving MCP in large graphs. These MCP algorithms (Rossi et al., 2014; Verma et al., 2015; San Segundo et al., 2016) heavily depend on the concept of the $k$-core (Seidman, 1983), which is a subgraph where all vertices have degree at least $k$, which can be computed in $O(m)$ ( $m$ is the number of edges) using bin sorting (Batagelj $\&$ Zaveršnik, 2003). We are not aware of any work using the $k$-core concept to develop MWCP algorithms, except implicitly by using an initial ordering of the vertices (Jiang, Li, \& Manyà, 2017). Moreover, an analogous concept in vertex-weighted graphs requires prohibitive space $(O(\bar{w} \cdot m)$, where $\bar{w}$ is the average weight of vertices) for bin sorting, and does not allow fast computation.

MWCP is more complicated than MCP and some powerful techniques for MCP are not applicable or ineffective for solving MWCP due to the vertex weights. This partly explains the fact that there are relatively fewer algorithms for MWCP. Some exact algorithms for MWCP come from and generalize previous BnB methods designed for MCP (Östergård, 1999; Kumlander, 2004). The MaxSAT-based method was also generalized to MWCP by Fang et al. (2014), resulting in an exact MWCP algorithm named MaxWClq. Jiang et al. (2017) proposed an exact BnB algorithm for MWCP called WLMC, which is especially designed for large scale graphs. WLMC incorporates two important techniques. The first is a preprocessing to derive an initial vertex ordering and to reduce the size of the graph, and the other is the incremental vertex-weight splitting to reduce the number of branches in the search space. In parallel with WLMC, Li et al. (2018) proposed a new upper bound for MWCP which is based on the notion of a weight cover. The idea of a weight cover 
is to compute a set of independent sets of the graph and define a weight function for each independent set so that the weight of each vertex of the graph is covered by such weight functions. This upper bound is used to develop a BnB-based exact algorithm named WC-MWC (Li et al., 2018). These two exact algorithms WLMC and WC-MWC achieve good results on large sparse graphs, and their performance is similar according to their experimental evaluation ( $\mathrm{Li}$ et al., 2018). More recently, Jiang et al. (2018) proposed a BnB algorithm that combines a novel two-stage MaxSAT reasoning approach with effective $\mathrm{BnB}$ techniques for large graphs, and the resulting algorithm is called TSM-MWC, which significantly outperforms WLMC on a broad range of large real-world benchmarks. Therefore, TSM-MWC is the state of the art in this direction.

For solving MWCP, many researchers are devoted to designing effective heuristic algorithms, aiming to find a good quality solution in short time. Massaro, Pelillo, and Bomze (2002) proposed a complementary pivoting algorithm based on the corresponding linear complementarity problem. Busygin (2006) presented a heuristic method using a nonlinear programming formulation for MWCP. A hybrid evolutionary approach was offered by Singh and Gupta (2006a). An efficient local search algorithm for MCP called Phased Local Search (PLS) was extended to MWCP (Pullan, 2008), which interleaves different modes of local search. A local search algorithm called MN/NT integrates a combined neighborhood and a dedicated tabu mechanism, and shows better performance than previous heuristic algorithms on a broad range of benchmarks (Wu, Hao, \& Glover, 2012). Afterwards, Wang, Cai, and Yin (2016) developed an improved local search algorithm called LSCC based on the configuration checking strategy and further improves MN/NT on a wide range of benchmarks. Based on LSCC, efficient local search MWCP algorithms for large graphs were developed. The LSCC+BMS algorithm improved LSCC by including a probabilistic heuristic called Best from Multiple Selection (BMS) (Cai, 2015), leading to much better performance on large graphs from the Network Data Repository. Fan et al. (2017) introduced the RRWL algorithm, another improved algorithm from LSCC, which incorporates restart, random walk and hash techniques, and shows better performance than LSCC+BMS. Besides the line of configuration-checking-based local search, Zhou, Hao, and Goëffon (2017) presented the generalized push operator for MWCP and then used this operator to develop two restarting tabu search algorithms ReTS1 and ReTS2. Nevertheless, ReTS1 and ReTS2 were mainly evaluated on medium- and small-sized graphs. Very recently, the authors of LSCC+BMS proposed the walk perturbation technique and several heuristics to diversify the search, resulting in an improved version named SCCWalk4L (Wang et al., 2020) for solving MWCP on large graphs. SCCWalk4L showed obviously better performance than previous heuristic algorithms on a broad range of large graphs (Wang et al., 2020), and thus represents the latest state of the art in heuristic MWCP algorithms for large graphs.

Further algorithms exist for solving MWCP, however, they are not feasible for solving large sparse graphs. San Segundo, Furini, and Artieda (2019) recently introduced an exact BnB algorithm for MWCP that uses a bitboard representation of the adjacency matrix, together with more advanced lower and upper bounds. However, as they use an adjacency matrix representation, their algorithm is not feasible for graphs with more than thousands of vertices. Of note here is that maximum weight independent set and minimum weight vertex cover solvers, many of which use data reductions, can also be used to solve MWCP (Lamm et al., 2019; Li et al., 2020; Cai et al., 2019). However, these algorithms would need to be run on the complement graph. For the graphs we consider here, the complement is dense and again infeasible to store in memory. 


\subsection{Contributions and Paper Organization}

In many applications, either the time limit for computation is very short or computational resources are limited; however, the input graphs may be very large. This motivates us to develop algorithms for solving MWCP for large graphs within a short time limit (e.g., 100 seconds). In this work, we focus on solving MWCP for large sparse graphs. For such graphs, we show that we can make use of the sparsity to develop efficient algorithms that can solve MWCP quickly.

As the main contribution, we propose a method that interleaves clique finding and graph reduction, which is very effective for solving large sparse graphs. In a graph reduction procedure, we reduce the size of the graph by removing vertices that are proved to be in no clique of maximum weight. For large sparse graphs, their size can be reduced significantly by using a clique of certain quality in hand as a lower bound together with effective upper bound functions. On the other hand, the remaining smaller graph presents smaller search space and the algorithm may find better cliques more easily, which can then be used to further reduce the graph. As far as we know, this is the first algorithm that interleaves clique finding and graph reduction. Some existing algorithms for MCP (Rossi et al., 2014; Verma et al., 2015; San Segundo et al., 2016) and MWCP (Jiang et al., 2017) reduce the graph in a preprocessing procedure, which is done just one time. Moreover, the proposed framework interleaving clique finding and graph reduction allows us to develop semiexact algorithms - the graph shrinks as the algorithm proceeds, and if the graph becomes empty, the found clique is proved to be optimal.

Besides the semi-exact algorithmic framework, we propose some ideas to make the method more efficient. The first two ideas are used in the clique finding phase:

- We propose a construct-and-cut algorithm for clique finding, in which an evaluation function is proposed for estimating the benefit of adding a vertex. The construct-and-cut method is proposed for the first time, which uses pruning techniques but differs from the BnB method.

- We also propose a dynamic version of the BMS heuristic, which is used in choosing a solution vertex.

The other two ideas are used in the graph reduction phase, including two novel upper bounds. The reduction rules based on these upper bounds significantly improve the ability of proving the optimality.

- We propose a branching-based upper bound, which makes use of the maximum weight neighboring vertex.

- We also propose a weighted-coloring-based upper bound, which relies on an algorithm to color the vertex-weighted graph.

Based on these ideas, we develop an algorithm called FastWClq ${ }^{1}$. To evaluate our algorithm, we consider two benchmarks of large graphs, the Network Data Repository benchmark (Rossi \& Ahmed, 2015) and the KONECT benchmark (Kunegis, 2013). Also, to study the performance on these graphs with different weight distributions, we not only test the instances with weights generated according to the 'mod200' method used in the literature (Pullan, 2009), but also test them

1. Note that although this name has been used to denote an early version of the algorithm in the conference paper, here FastWClq refers to the final version as presented in this work. 
with weights generated according to a normal distribution. Finally, we obtain four benchmarks for our experiments.

We compare our FastWClq algorithm with state-of-the-art algorithms for solving very large MWCP instances, including both exact and heuristic algorithms. Experiments show that FastWClq significantly outperforms previous algorithms in terms of the solution quality and run time. Further, as a semi-exact algorithm, FastWClq proves the optimality of its solutions for more than half of the challenging large graphs with more than 100000 vertices, achieving similar (yet worse) performance with the state-of-the-art exact MWCP algorithm TSM-MWC in terms of the proving ability.

Note that an early version of FastWClq has been published in a conference paper (Cai \& Lin, 2016). We describe the new contributions in this article below. We optimize the framework of FastWClq and propose two new ideas to improve FastWClq, including a clique-improving method during the clique finding phase and a weighted-coloring-based upper bound during the reduction phase. In addition, we add more experiments to evaluate performance of FastWClq, and compare with the latest state-of-the-art algorithms.

In the next section, we introduce some necessary background knowledge. Then, we describe the framework of the semi-exact method in Section 3. The details of the clique finding phase and the graph reduction phase are presented in Section 4 and 5 respectively. Experimental evaluations of our algorithm, FastWClq, are presented in Section 6. Finally, we give some concluding remarks and outline the future work in Section 7.

\section{Preliminaries}

Let $G=(V, E)$ be an undirected graph where $V=\left\{v_{1}, v_{2}, \ldots, v_{n}\right\}$ is the set of vertices and $E \subset$ $\{\{u, v\} \mid u, v \in V\}$ is the set of edges in $G$. We denote their cardinalities by $n=|V|$ and $m=|E|$. We use $V(G)$ and $E(G)$ to denote the vertex set and the edge set of graph $G$. A vertex-weighted undirected graph is an undirected graph $G=(V, E)$ combined with a mapping $w: V \mapsto \mathbb{N}$ (where $\mathbb{N}$ is the set of natural numbers) so that each vertex $v \in V$ is associated with a natural number $w(v)$ as its weight. We use a triple to denote a vertex-weighted graph, i.e., $G=(V, E, w)$. For a subset $S \subseteq V$, we let $G[S]$ denote the subgraph induced by $S$, which is formed from $S$ and all the edges $\{\{u, v\} \in E \mid u, v \in S\}$ connecting pairs of vertices in $S$. The weight of $S$ is $w(S)=\sum_{v \in S} w(v)$. The (open) neighborhood of a vertex $v$ is $N(v)=\{u \in V \mid\{u, v\} \in E\}$, and we denote the closed neighborhood by $N[v]=N(v) \cup\{v\}$. The degree of $v$ is $d(v)=|N(v)|$.

Given a graph $G$, a clique $C \subseteq V$ is a set of pairwise adjacent vertices, while an independent set $I$ is a set of pairwise nonadjacent vertices. A clique or independent set is maximal if it is not included in a larger clique or independent set. The maximum clique problem $(\mathrm{MCP})$ is to find a clique of maximum cardinality in a graph, and the maximum weight clique problem (MWCP) is to find a clique of the maximum weight in a vertex-weighted graph. We say a vertex is "bad" if it is proved to be in no optimal solution.

For a graph, a proper vertex coloring is an assignment of colors to all vertices of the graph such that no two adjacent vertices share the same color. Equivalently, a proper vertex coloring of a graph $G$ is a partition $\left\{A_{1}, A_{2}, \ldots, A_{k}\right\}$ of the vertex set $V(G)$ into independent sets. Under a proper vertex coloring, the set of vertices for a color forms an independent set. Therefore, any clique can contain at most one vertex from one color. This can be used to compute an upper bound for MCP and MWCP. 


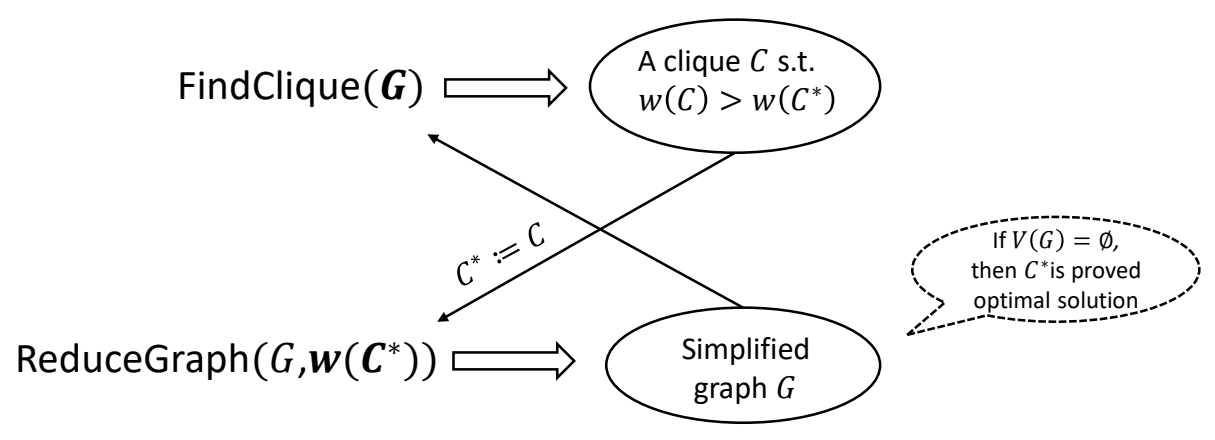

Figure 1. A semi-exact method for MWCP

The BMS (Best from Multiple Selection) heuristic by Cai (2015) is a sampling technique, which is used to choose a good-quality element from a large set. It randomly picks $k$ elements and returns the best one with respect to some criterion. We use a dynamic BMS heuristic in our algorithm.

\section{A Semi-Exact Method for MWCP: The Framework}

In this section, we introduce the framework of our semi-exact method for solving MWCP, which interleaves clique finding and graph reduction. The algorithmic framework is illustrated in Figure 1.

This semi-exact method is composed of two sub-algorithms, a clique finding algorithm and a graph reduction algorithm. The clique finding algorithm aims to find a larger-weight clique than the best found clique $C^{*}$; once such a clique is found, $C^{*}$ is updated to this new clique. As $w\left(C^{*}\right)$ is a lower bound on the optimal clique weight, when $w\left(C^{*}\right)$ is updated to a larger value, a tighter lower bound is obtained, and then the graph reduction algorithm is called to further simplify the graph. The graph reduction algorithm aims to simplify the graph by detecting "bad" vertices (i.e. those vertices that cannot be in any clique of maximum weight) and removing as many of them as possible. Additionally, if the graph becomes empty after reduction, then the best found solution $C^{*}$ is proved to be optimal. Hence, our method is a semi-exact method.

Based on this framework, we develop an algorithm called FastWClq. The pseudocode of FastWClq is shown in Algorithm 1. After the initialization, the algorithm executes a main loop until a limited time is reached, or an exact solution is found and proved. In the main loop, FastWClq works by interleaving the two sub-algorithms, as shown in Figure 1. The algorithm for clique finding in FastWClq adopts a construct-and-cut technique. In each iteration (line 4), it constructs a maximal clique step by step. Also, the construction procedure may be cut before reaching a maximal clique, using pruning techniques by computing upper bounds. We introduce the details of the two sub-algorithms in the next two sections.

\section{Clique Finding}

In this section, we describe our algorithm for clique finding. We propose a novel method called construct-and-cut for quickly finding a high quality clique. Also, when a clique of higher weight (compared to the best found one) is located, we further improve the clique by trying possible local modifications. 


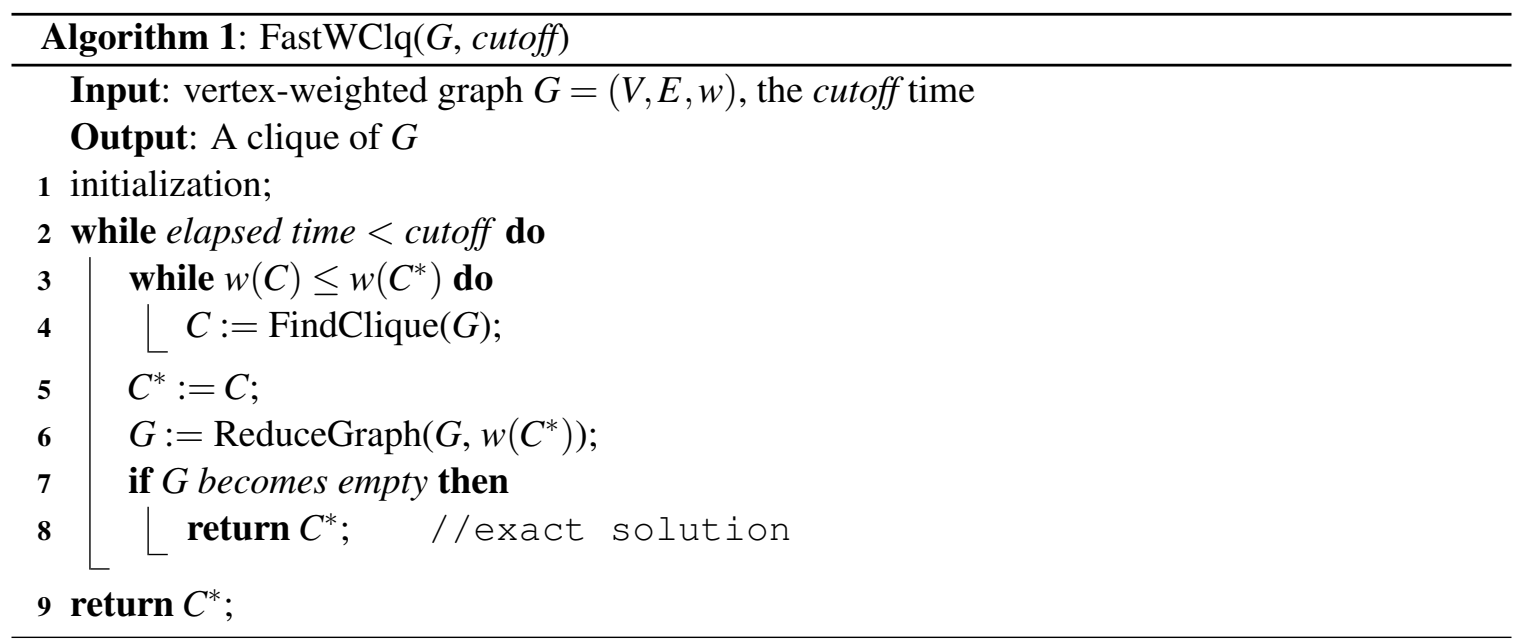

Before going to the details of the construct-and-cut algorithm, let us first introduce some notation and definitions.

- $C$ : the current clique under construction.

- StartSet: the set containing candidate vertices which can serve as a starting vertex to construct a clique. In the beginning of the algorithm, StartSet is initialized as $V(G)$.

- CandSet $=\bigcap_{v \in C} N(v)$, i.e., each vertex in CandSet is adjacent to all vertices in $C$. This is the set of candidate vertices that can be added to extend the current clique.

- The effective neighborhood of vertex $v$ : is defined as $N(v) \cap$ CandSet. The concept is very important, as $w(N(v) \cap$ CandSet $)$ is used in both pruning a construction procedure and evaluating the quality of candidate vertices.

In our clique construction procedure (Algorithm 2), the algorithm first pops a random vertex from StartSet to serve as the starting vertex from which a clique will be extended, if StartSet is not empty (line 4). If StartSet becomes empty, which means all vertices have been used as the starting vertex, then another round of clique construction begins by resetting StartSet to $V(G)$, and we adjust our strategy parameter (lines 1-3). After the starting vertex $u$ is chosen, the clique is initialized with the vertex, and CandSet is initialized as $N(u)$ (lines 5-6). Then the clique is extended iteratively by each time adding a vertex $v \in$ CandSet, until CandSet becomes empty (lines 7-12). Also, we use a cost-effective upper bound to prune the procedure (line 9) - the construction procedure is cut before the normal termination. Obviously, $w(C)+w(v)+w(N(v) \cap$ CandSet $)$ is an upper bound on weight of any clique extended from $C$ by adding $v$ and more vertices. We would like to note that, the construct-and-cut method has recently been used to generate initial assignments for the Boolean Satisfiability (SAT) problem (Cai, Luo, Zhang, \& Zhang, 2021), which is parallel to this work.

\subsection{Choosing a Solution Vertex}

An important component in FindClique is the procedure ChooseSolutionVertex (Algorithm 3), which selects a vertex from CandSet to extend the current clique. We call such a vertex a solu- 

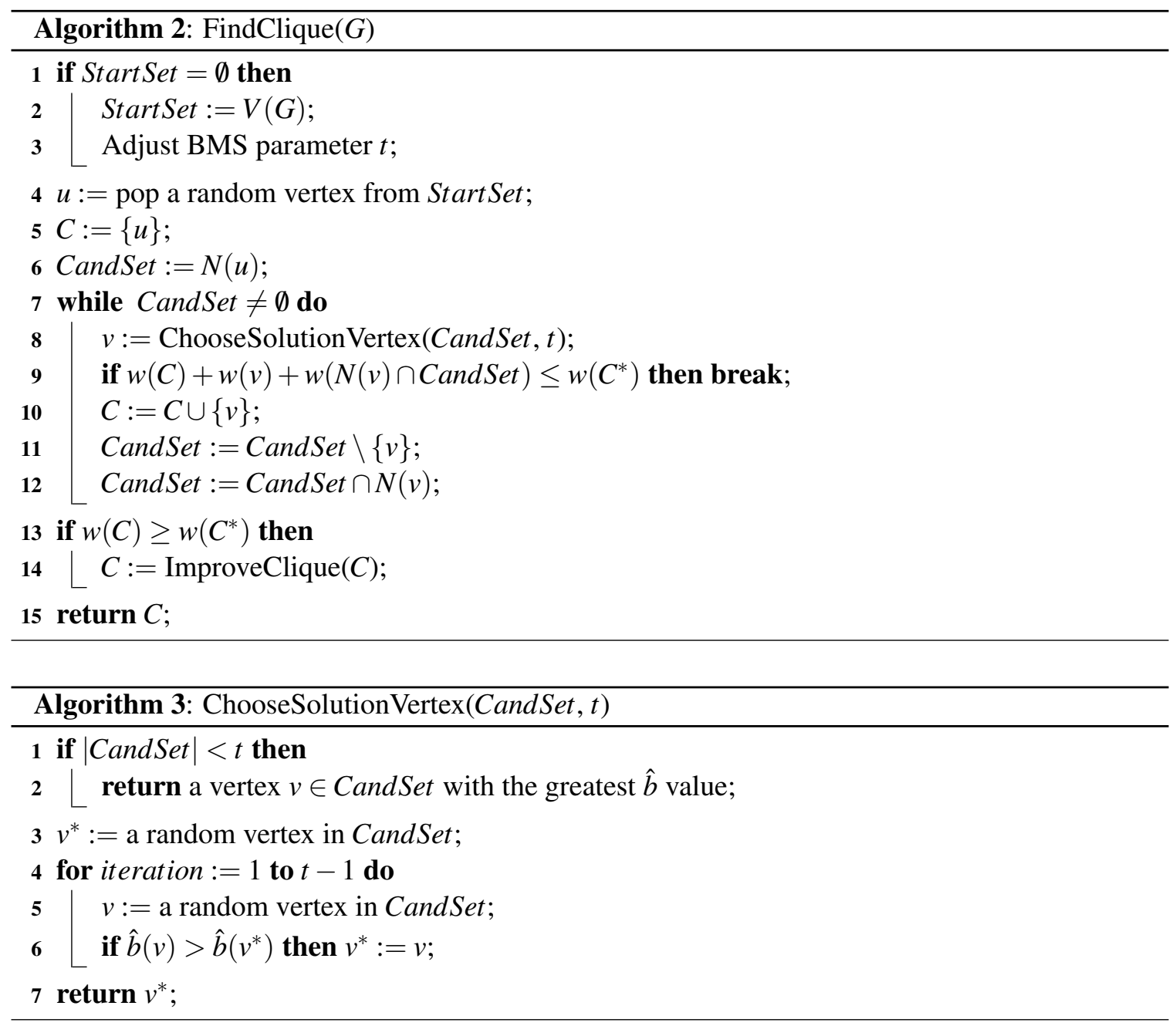

tion vertex. To this end, we propose a novel function to estimate the benefit of vertices, and a dynamic BMS heuristic to choose a solution vertex.

Benefit Estimation Function. For choosing a vertex to add into the current clique, we need to estimate the benefit of adding a vertex, and then we choose the one with the best estimated benefit. We define the benefit of adding a vertex $v$ as benefit $(v)=w\left(C_{f}\right)-w(C)$, where $C_{f}$ is the final clique grown from $C \cup\{v\}$ by the construction procedure.

An ideal strategy is to pick the vertex with the best benefit at each iteration to extend the clique. However, we cannot know the true benefit value of a vertex until we finish the construction procedure. In order to compare candidate vertices at the current iteration, we propose a function to estimate the benefit of adding a vertex. The function is based on two considerations:

1. If a candidate vertex $v$ is added into the clique $C$, the weight of $C$ is increased by $w(v)$, which is a trivial lower bound of benefit $(v)$.

2. Suppose a candidate vertex $v$ is selected to be added into the clique $C$. The best possible weighted clique grown from $C \cup\{v\}$ is $C \cup\{v\} \cup(N(v) \cap$ CandSet $)$, for which the weight is 
$w(C)+w(v)+w(N(v) \cap$ CandSet $)$. Thus, an upper bound of benefit $(v)$ is $w(v)+w(N(v) \cap$ CandSet).

An estimation function should take into account both the lower bound and upper bound of benefit $(v)$. A simple and intuitive function which embodies this principle is to take the average over these two bounds:

$$
\begin{aligned}
\hat{b}(v) & =\frac{w(v)+w(v)+w(N(v) \cap \text { CandSet })}{2} \\
& =w(v)+w(N(v) \cap \text { CandSet }) / 2 .
\end{aligned}
$$

Dynamic BMS Heuristic. We choose the solution vertex based on the $\hat{b}$ values of candidates, according to a dynamic BMS heuristic. The original BMS heuristic is a probabilistic strategy which returns the best element from multiple samples. Cai (2015) theoretically showed that BMS can approximate the best selection strategy very well in $O(1)$ time. The probability that the BMS heuristic chooses a vertex whose $\hat{b}(v)$ value is better than $\rho$ of the vertices in $\operatorname{CandSet}$ is $\operatorname{Pr}(E)>1-\rho^{t}$. Another advantage of the BMS heuristic is that we can control the greediness of the algorithm by adjusting the parameter $t$. However, this has not been exploited previously, and previous algorithms with BMS adopt a static parameter, that is, the number of samplings $t$ stays the same (Cai, 2015; Wang et al., 2016).

In general, a greater $t$ value indicates a greater greediness and more computation time. Based on this observation, we propose a dynamic BMS heuristic. In our algorithm, we start from a small $t$ value (denoted as $t_{0}$ ), so that the algorithm works fast. Whenever StartSet becomes empty, which means we do not find a better clique with this $t$ value, we double $t$ (i.e., $t:=2 t$ ), with a upper bound limitation $t_{\max }$, to make the algorithm construct cliques in a greedier way.

\subsection{Improving the Clique}

In the clique construction procedure, if a better clique $C$ (compared to the best-found one) is obtained, we try to further improve it by examining the possible improvement with respect to each vertex in the clique. Specifically, for each vertex $v \in C$, we examine whether the following modification results in a larger-weight clique:

We remove $v$ from $C$, and then CandSet is set to the common neighborhood of $C \backslash\{v\}$ accordingly. Then, find a maximum weight clique $C_{v}^{\prime}$ from $C$ and $S e t$, using a branch-and-bound method (the bound is given by weighted coloring, which will be introduced in Section 5.2 in detail). If $w\left(C_{v}^{\prime}\right)>w(v)$, then a better clique $(C \backslash\{v\}) \cup C_{v}^{\prime}$ is found and we update $C$ accordingly. Otherwise, $C$ remains unchanged.

Such examinations of possible improvements are executed for all vertices $v \in C$, and $C$ is updated whenever a modification with improvement is identified. This procedure terminates when all vertices $v \in C$ are examined. As the size of CandSet is relatively small, this procedure is very fast.

\section{Graph Reduction}

By applying sound data reduction rules (which usually depend on a clique in hand), a graph can be reduced to a (sometimes significantly) smaller graph while keeping the optimal solution. This is desirable as algorithms can solve the original instance by solving a smaller and easier one. Many existing reduction rules for weighted problems rely on local structure (Lamm et al., 2019; Li et al., 2020), whereas our data reduction rules exploit lower bounds and upper bounds to reduce the graph 
globally. While the lower bound is simply the weight of the current best clique, much effort is devoted to designing upper bound functions. In this section, we introduce a graph reduction algorithm, which relies on three upper bounds: a trivial bound, a branching-based bound and a coloring-based bound; the latter two bounds are proposed in this work.

Definition 1 (A Generic Upper Bound, UB). Given a vertex-weighted graph $G=(V, E, w)$ and a vertex $v \in V(G)$, we let $U B(v)$ denote an (integer) upper bound on the weight of any clique containing $v$. That is, $U B(v) \geq \max \{w(C) \mid C$ is a clique of $G, v \in C\}$.

Now, we consider the below reduction rule, which is a general rule and should be used with the help of a lower bound and a upper bound.

General Reduction Rule. Given a vertex-weighted graph $G=(V, E, w)$ and a clique $C_{0}$ in $G$, $\forall v \in V(G)$, if there is an upper bound $U B(v)$ such that $U B(v) \leq w\left(C_{0}\right)$, then delete $v$ and its incident edges from $G$.

The above rule indeed represents a family of reduction rules. In order to obtain an applicable concrete rule, we need to specify the upper bound function and the input clique. We use the notation $\operatorname{Rule}\left(U B, C_{0}\right)$ to denote a concrete rule where $U B$ is the upper bound function and $C_{0}$ is the input clique.

Proposition 1 (Soundness of the General Reduction Rule). Let $G$ be a vertex-weighted graph, $G^{\prime}$ the resulting graph by applying Rule $\left(U B, C_{0}\right)$ on $G$, and let $w^{*}$ be the weight of a maximum weight clique of graph $G$, and $C_{G^{\prime}}^{*}$ the maximum weight clique of $G^{\prime}$. Then, $w^{*}=\max \left\{w\left(C_{0}\right), w\left(C_{G^{\prime}}^{*}\right)\right\}$.

Proof. If $w\left(C_{0}\right)=w^{*}$, then the proposition obviously holds. Now we consider the case $w\left(C_{0}\right)<w^{*}$. For graph $G$ and a vertex $v \in V(G)$, let $C_{v}^{*}$ be a clique with the maximum weight among cliques containing vertex $v$. If a function $U B$ satisfies Definition 1 , we have $w\left(C_{v}^{*}\right) \leq U B(v)$. On the other hand, any vertex deleted by Rule $\left(U B, C_{0}\right)$ satisfies $U B(v) \leq w\left(C_{0}\right)$, and thus $w\left(C_{v}^{*}\right) \leq U B(v) \leq$ $w\left(C_{0}\right)<w^{*}$, meaning that $v$ cannot be contained in any clique with weight $w^{*}$. Thus, any vertex that is in a clique with weight $w^{*}$ remains in $G^{\prime}$, so $w^{*}=w\left(C_{G^{\prime}}^{*}\right)$.

The above proposition shows that any specialization of the general reduction rule (for any valid upper bound) is sound with respect to keeping the optimal solution of the instance. Additionally, the proposition leads to the following corollary.

Corollary 1. Let $G^{\prime}$ be the resulting graph after applying Rule $\left(U B, C_{0}\right)$ on vertex-weighted graph $G$, if $V\left(G^{\prime}\right)=\emptyset$, then $C_{0}$ is a maximum weight clique of $G$.

\subsection{The Trivial Upper Bound and a Branching-Based Upper Bound}

Given a clique in hand (found by a clique-finding algorithm), in order to apply reduction rules, the focus is how to compute an upper bound. Since any clique grown from vertex $v$ can only contain vertices in $N(v)$, a trivial upper bound function is

$$
U B_{0}(v)=w(N[v]) .
$$

A tighter bound can be obtained by considering a branching-based upper bound function. For a vertex $v$, we consider its neighboring vertex with the maximum weight (denoted as $n^{*}$ ). The idea is 
that, for any clique $C$ containing $v$, it either contains $n^{*}$ or it does not. For either case, we can have a tighter upper bound than $U B_{0}(v)$, and finally we get the larger (worse) one as the upper bound. We divide the cases on $n^{*}$ in order to balance the bounds of the two cases. Formally, we propose a branching-based upper bound as follows:

$$
\begin{aligned}
U B_{1}(v) & =\max \left\{w(N[v])-w\left(n^{*}\right), w(v)+w\left(n^{*}\right)+w\left(N(v) \cap N\left(n^{*}\right)\right)\right\} \\
& =w(v)+\max \left\{w(N(v))-w\left(n^{*}\right), w\left(n^{*}\right)+w\left(N(v) \cap N\left(n^{*}\right)\right)\right\} .
\end{aligned}
$$

This upper bound can be easily understood: for any clique containing $v$, if it does not contain $n^{*}$, then an upper bound on the weight of the clique is $w(N[v])-w\left(n^{*}\right)$; if the clique contains $n^{*}$, then, besides $v$ and $n^{*}$, the clique can only contain those vertices that are adjacent to both $v$ and $n^{*}$. Thus, an upper bound on the weight of the clique is $w(v)+w\left(n^{*}\right)+w\left(N(v) \cap N\left(n^{*}\right)\right)$. Finally, the worst case can be used as an upper bound on the weight of any clique containing $v$.

Note that we use an adjacency list instead of an adjacency matrix representation, to ensure that the graph fits into memory. So, checking whether a vertex $y \in N(v)$ is in $N\left(n^{*}\right)$, i.e., whether $y$ and $n^{*}$ are neighbors, requires $O\left(\min \left\{d(y), d\left(n^{*}\right)\right\}\right)$ time. Note that a straightforward, but inefficient, way to compute $N(v) \cap N\left(n^{*}\right)$ is by checking for each vertex $y \in N(v)$ if $y \in N\left(n^{*}\right)$-which is quadratic in the degree. Rather than use the above implementation, we compute $N(v) \cap N\left(n^{*}\right)$ in linear time with two scans on the smaller set and one on the larger one, using indicators.

\subsection{Improving Branching-Based Upper Bound by Weighted Coloring}

The branch-based upper bound $U B_{1}$ can be improved by further using coloring on each situation, leading to a tighter upper bound. We describe this new upper bound in this subsection.

For a vertex-weighted graph $G$, suppose we have a proper vertex coloring $A_{1}, A_{2}, \ldots, A_{k}$ of the graph, where $A_{i}$ is the set of vertices with color $i$, then an upper bound on the weight of the maximum clique weight is as follows:

$$
U B_{c}(G)=\sum_{i=1}^{k} \max _{u \in A_{i}}\{w(u)\} .
$$

This is easy to understand, as vertices with the same color composes an independent set and thus at most one of them can be selected in a valid clique. By integrating this weighted-coloring-based upper bound, we can further make the branching-based upper bound tighter:

$$
U B_{2}(v)=w(v)+\max \left\{U B_{c}\left(G\left[N(v) \backslash\left\{n^{*}\right\}\right]\right), w\left(n^{*}\right)+U B_{c}\left(G\left[N(v) \cap N\left(n^{*}\right)\right]\right)\right\} .
$$

The quality of the $U B_{2}$ upper bound depends on the coloring solution to obtain the $U B_{c}$ values. To leverage the power of this upper bound function, we need to compute a $U B_{c}$ value as small as possible. This indeed is a variant of the vertex coloring problem, which is referred to as the weighted-vertex coloring problem (Demange et al., 2002) or max-coloring problem (Hsu \& Chang, 2016).

Definition 2 (Weighted-Coloring Problem). The max-coloring problem is, for a given vertex-weighted graph, to find a proper vertex coloring $A_{1}, A_{2}, \ldots, A_{k}$ which minimizes $\sum_{i=1}^{k} \max _{u \in A_{i}}\{w(u)\}$.

In this work, since the algorithm for solving Weighted-Coloring is called many times, we employ a light-weight algorithm, which is described as follows. Sort the vertices in a weight decreasing 
order, breaking ties by vertex degree. According to this order, the vertices are colored one by one. For each vertex $v$, suppose that $k$ is the number of colors have been used so far, we find a color with the lowest value in $[1, k]$ that gives a proper coloring for $v$. If no such proper coloring exists, we color $v$ with a new color $k+1$.

\subsection{The Graph Reduction Algorithm}

The graph reduction algorithm is depicted in Algorithm 4. All the three upper bounds are used. $U B_{0}$ requires little overhead, while $U B_{1}$ and $U B_{2}$ require more computation time but are tighter. Therefore, when considering a vertex, we first use the $U B_{0}$-based reduction rule, and if this cannot delete the vertex then we apply the rule based on $U B_{1}$ and $U B_{2}{ }^{2}$.

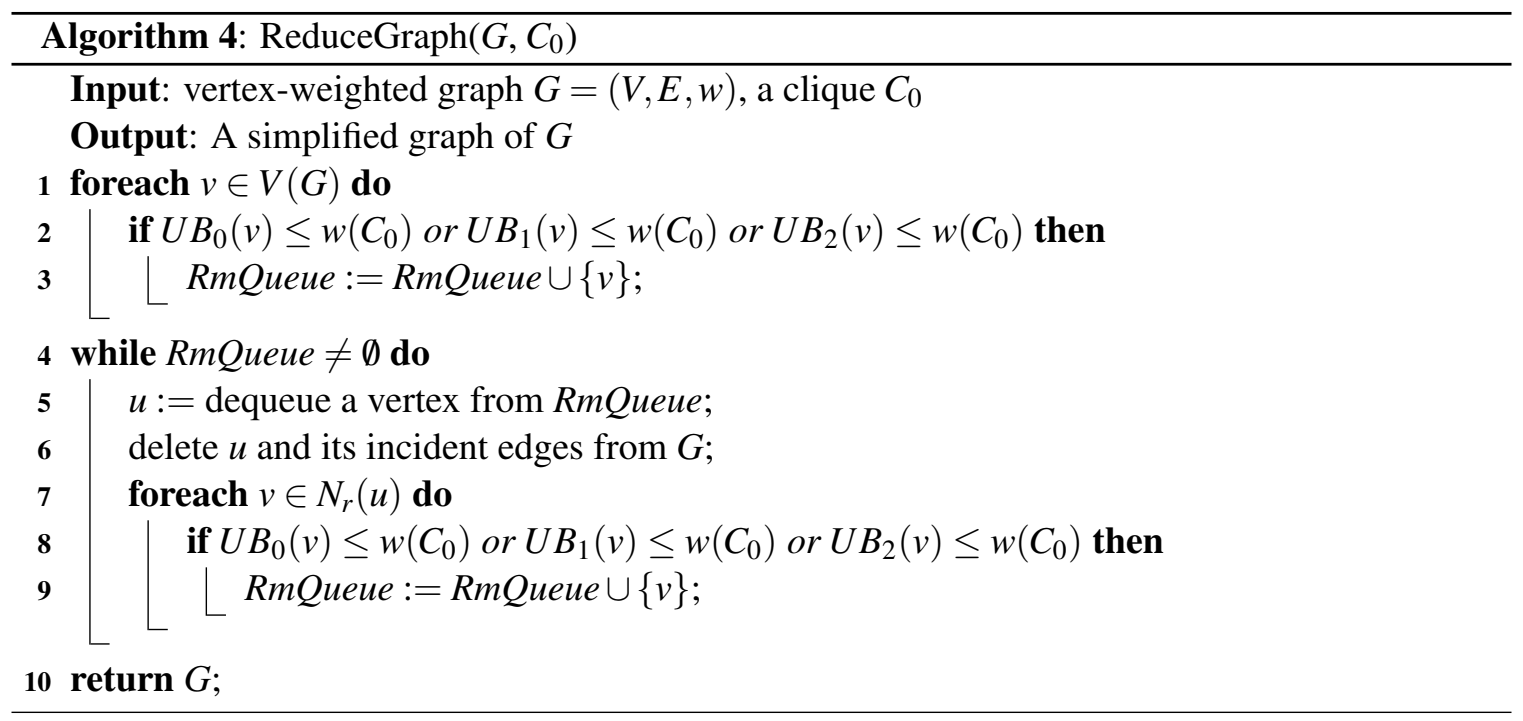

Our reduction algorithm works in an iterative fashion, with a queue called RmQueue which contains vertices to be deleted. In the beginning, the algorithm enqueues into RmQueue all vertices satisfying at least one of the reduction rules. Then, a loop is carried out until RmQueue becomes empty. Each iteration of the loop dequeues a vertex $u$ from RmQueue, and deletes $u$ and all its incident edges from $G$. After a vertex $u$ is deleted, we check its remained neighborhood $N_{r}(u)$ (the set containing all neighbors of $u$ that have not been removed from the graph yet), and enqueue to RmQueue all vertices in $N_{r}(u)$ that satisfy at least one of the reduction rules.

According to Corollary 1, if the ReduceGraph algorithm returns an empty graph, that means the clique found is a maximum weight clique of the input graph. However, there are cases that FastWClq finds a maximum weight clique but ReduceGraph cannot reduce the graph to empty, because the reduction rules are incomplete ${ }^{3}$.

2. In practice, a trick to accelerate the procedure (slightly) for large-sized graphs is to first use $U B_{0}$ to reduce the graph to a certain size, after which $U B_{1}$ and $U B_{2}$ are used.

3. Note that if this weren't the case and the reduction rules always resulted in an empty graph, then this would give a polynomial time algorithm for the decision variant of MWCP and $\mathrm{P}=\mathrm{NP}$. 


\section{Experimental Evaluation}

In this section, we carry out experiments to evaluate our proposed algorithm, FastWClq, on a broad range of large sparse real-world graphs. First, we introduce the benchmarks used in our experiments, and present some preliminaries about our experimental evaluation. We then compare FastWClq with state-of-the-art exact and heuristic algorithms. Our experiments show that FastWClq performs very well on a considerable portion of the selected instances.

\subsection{Benchmarks}

For our experiments, we collect two benchmarks of large sparse graphs, which are originally unweighted, and then we generate weights for vertices in these graphs. The two benchmarks of large sparse graphs are introduced as follows:

- Network Data Repository: This benchmark was downloaded from the Network Data Repository ${ }^{4}$ (Rossi \& Ahmed, 2015). We consider the 187 graphs used in testing WLMC (Jiang et al., 2017), and select the graphs with at least 100000 vertices and 1000000 edges. Some graphs in this benchmark are bipartite graphs, and we choose to ignore them. All these graphs are generated from real-world applications, which can be grouped into eleven classes, including biological networks, collaboration networks, interaction networks, infrastructure networks, Amazon recommendation networks, scientific computation networks, social networks, technological networks, and web-crawl graphs. Many of these real-world graphs have millions of vertices and tens of millions of edges, and each graph is quite sparse. Calculating the density $m /\left(\begin{array}{l}n \\ 2\end{array}\right)$ of each graph, the average density of these graphs is 0.00859 , while the maximum density is 0.347 . We also calculate the average degree $2 m / n$ for each graph; over all graphs, this figure is 26.15 on average, while the maximum is 181.19 .

- Koblenz Network Collection: This benchmark was downloaded from the Koblenz Network Collection (KONECT) ${ }^{5}$ (Kunegis, 2013), which was collected by the Institute of Web Science and Technologies at the University of Koblenz-Landau in order to perform research in network science and related fields. The networks of KONECT cover many diverse areas such as social networks, hyperlink networks, authorship networks, physical networks, interaction networks, and communication networks. We downloaded all the graphs from KONECT and converted them into undirected graphs, except the bipartite graphs. These large graphs are also quite sparse. The average density is 0.000916 , while the maximum one is 0.022332 . The average degree is 18.25 , while the maximum one is 211.44 .

In our experiments, we focus on the large graphs, so we select those graphs with at least 100000 vertices from each benchmark, resulting in 65 graphs from Network Data Repository and 80 graphs from KONECT benchmark. The sizes of these large graphs are given in Tables 1 and 2.

To obtain the corresponding vertex-weighted graphs, we generate vertex weights to the graphs of the above two benchmarks in two different ways.

- v_mod_200: For the $i^{t h}$ vertex $v_{i}, w\left(v_{i}\right)=(i \bmod 200)+1$.

4. http://www.networkrepository.com

5. http://konect.uni-koblenz.de 
Table 1. Details of graphs with at least 100000 vertices from Network Data Repository

\begin{tabular}{lrr}
\hline Instance & $|V|$ & $|E|$ \\
\hline bn-human-BNU_1_0025865_session_1-bg & 1398408 & 42296922 \\
bn-human-BNU_1_0025865_session_2-bg & 1717207 & 22855526 \\
ca-coauthors-dblp & 540486 & 15245729 \\
ca-dblp-2012 & 317080 & 1049866 \\
ca-hollywood-2009 & 1069126 & 56306653 \\
channel-500x100x100-b050 & 4802000 & 42681372 \\
dbpedia-link & 11621692 & 78621046 \\
delaunay_n22 & 4194304 & 12582869 \\
delaunay_n23 & 8388608 & 25165784 \\
delaunay_n24 & 16777216 & 50331601 \\
friendster & 8658744 & 45671471 \\
hugebubbles-00020 & 21198119 & 31790179 \\
hugetrace-00010 & 12057441 & 18082179 \\
hugetrace-00020 & 16002413 & 23998813 \\
inf-europe_osm & 50912018 & 54054660 \\
inf-germany_osm & 11548845 & 12369181 \\
inf-road-usa & 23947347 & 28854312 \\
inf-roadNet-CA & 1957027 & 2760388 \\
inf-roadNet-PA & 1087562 & 1541514 \\
rec-dating & 168792 & 17351416 \\
rec-epinions & 755761 & 13396042 \\
rec-libimseti-dir & 220970 & 17233144 \\
rgg_n_2_23_s0 & 8388608 & 63501393 \\
rgg_n_2_24_s0 & 16777216 & 132557200 \\
rt-retweet-crawl & 1112702 & 2278852 \\
sc-ldoor & 952203 & 20770807 \\
sc-msdoor & 415863 & 9378650 \\
sc-pwtk & 217891 & 5653221 \\
sc-rel9 & 5921786 & 23667162 \\
sc-shipsec1 & 140385 & 1707759 \\
sc-shipsec5 & 179104 & 2200076 \\
soc-FourSquare & 639014 & 3214986 \\
soc-LiveMocha & 104103 & 2193083 \\
\hline & & \\
& &
\end{tabular}

\begin{tabular}{lrr}
\hline Instance & $|V|$ & $|E|$ \\
\hline soc-buzznet & 101163 & 2763066 \\
soc-delicious & 536108 & 1365961 \\
soc-digg & 770799 & 5907132 \\
soc-dogster & 426820 & 8543549 \\
soc-flickr-und & 1715255 & 15555041 \\
soc-flickr & 513969 & 3190452 \\
soc-flixster & 2523386 & 7918801 \\
soc-lastfm & 1191805 & 4519330 \\
soc-livejournal-user-groups & 7489073 & 112305407 \\
soc-livejournal & 4033137 & 27933062 \\
soc-ljournal-2008 & 5363186 & 49514271 \\
soc-orkut-dir & 3072441 & 117185083 \\
soc-orkut & 2997166 & 106349209 \\
soc-pokec & 1632803 & 22301964 \\
soc-sinaweibo & 58655849 & 261321033 \\
soc-twitter-higgs & 456631 & 12508442 \\
soc-youtube-snap & 1134890 & 2987624 \\
soc-youtube & 495957 & 1936748 \\
socfb-A-anon & 3097165 & 23667394 \\
socfb-B-anon & 2937612 & 20959854 \\
socfb-uci-uni & 58790782 & 92208195 \\
tech-as-skitter & 1694616 & 11094209 \\
tech-ip & 2250498 & 21643497 \\
twitter_mpi & 9862152 & 99940317 \\
web-arabic-2005 & 163598 & 1747269 \\
web-baidu-baike & 2141300 & 17014946 \\
web-it-2004 & 509338 & 7178413 \\
web-uk-2005 & 129632 & 11744049 \\
web-wikipedia-growth & 1870709 & 36532531 \\
web-wikipedia2009 & 1864433 & 4507315 \\
web-wikipedia_link & 2936413 & 86754664 \\
wikipedia_link_en & 27154756 & 31024475 \\
& & \\
& & \\
& &
\end{tabular}


Table 2. Details of graphs with at least 100000 vertices from KONECT benchmark

\begin{tabular}{lrr}
\hline Instance & $|V|$ & $|E|$ \\
\hline actor-collaboration & 382219 & 15038083 \\
amazon0601 & 403394 & 2443408 \\
as-skitter & 1696415 & 11095298 \\
citeseer & 384413 & 1736145 \\
com-amazon & 334863 & 925872 \\
com-dblp & 317080 & 1049866 \\
com-youtube & 1134890 & 2987624 \\
dbpedia-all & 3966895 & 12610982 \\
dbpedia-link & 18268991 & 126890209 \\
digg-friends & 1923999 & 3192495 \\
douban & 154908 & 327162 \\
elec & 105144 & 200184 \\
email-EuAll & 265214 & 364481 \\
enron & 307636 & 517819 \\
epinions & 132768 & 713088 \\
facebook-wosn-links & 397655 & 1150959 \\
facebook-wosn-wall & 914890 & 1051350 \\
flickrEdges & 105938 & 2316948 \\
flickr-growth & 2303059 & 22838410 \\
flickr-links & 1715254 & 15551249 \\
flixster & 2523386 & 7918801 \\
hyves & 1402673 & 2777419 \\
lasagne-yahoo & 653260 & 2931698 \\
libimseti & 220970 & 10131013 \\
link-dynamic-dewiki & 19521519 & 61151432 \\
link-dynamic-frwiki & 14371085 & 44187494 \\
link-dynamic-itwiki & 8549267 & 28858941 \\
link-dynamic-nlwiki & 6126550 & 18873552 \\
link-dynamic-plwiki & 6231281 & 20768067 \\
link-dynamic-simplewiki & 380731 & 1137693 \\
livejournal-links & 5204175 & 48709621 \\
livemocha & 104103 & 2193083 \\
lkml-reply & 909627 & 1041696 \\
loc-gowalla_edges & 196591 & 950327 \\
munmun_digg_reply & 114340 & 169097 \\
munmun_twitter_social & 465017 & 833540 \\
orkut-links & 3072441 & 117184899 \\
patentcite & 3774768 & 16518947 \\
petster-carnivore & 623766 & 15695166 \\
petster-cat-uniq & 149700 & 5448197 \\
\hline
\end{tabular}

\begin{tabular}{lrr}
\hline Instance & $|V|$ & $|E|$ \\
\hline petster-dog-uniq & 426820 & 8543549 \\
roadNet-CA & 1965206 & 2766607 \\
roadNet-PA & 1088092 & 1541898 \\
roadNet-TX & 1379917 & 1921660 \\
slashdot-threads & 141428 & 206918 \\
soc-LiveJournal1 & 4846609 & 42851237 \\
soc-pokec-relationships & 1632803 & 22301964 \\
trec-wt10g & 1601787 & 6679248 \\
web-BerkStan & 685230 & 6649470 \\
web-Google & 875713 & 4322051 \\
web-NotreDame & 325729 & 1090108 \\
web-Stanford & 281903 & 1992636 \\
wikipedia-growth & 1872907 & 36534729 \\
wikipedia_link_de & 3225565 & 65759634 \\
wikipedia_link_en & 12150976 & 288257813 \\
wikipedia_link_fr & 3023165 & 83455052 \\
wikipedia_link_it & 1865965 & 68022541 \\
wikipedia_link_ja & 1610638 & 56231610 \\
wikipedia_link_pl & 1529135 & 42188631 \\
wikipedia_link_pt & 1603222 & 38633429 \\
wikipedia_link_ru & 2853118 & 63058425 \\
wikisigned-k2 & 138593 & 482888 \\
wiki-Talk & 2394385 & 4659565 \\
wiki_talk_ar & 2868931 & 3307474 \\
wiki_talk_de & 6757916 & 7744939 \\
wiki_talk_en & 26373842 & 31532851 \\
wiki_talk_es & 2661000 & 3228049 \\
wiki_talk_fr & 5637678 & 6484425 \\
wiki_talk_it & 3671312 & 4309561 \\
wiki_talk_nl & 1615381 & 1900124 \\
wiki_talk_pt & 2694832 & 3533288 \\
wiki_talk_ru & 2470379 & 2862384 \\
wiki_talk_zh & 3175241 & 3644578 \\
wordnet-words & 146005 & 656999 \\
youtube-links & 1138494 & 2990287 \\
youtube-u-growth & 3223788 & 9375577 \\
zhishi-baidu-internallink & 2141300 & 17014946 \\
zhishi-baidu-relatedpages & 415641 & 2374044 \\
zhishi-hudong-internallink & 1984484 & 14428382 \\
zhishi-hudong-relatedpages & 2452715 & 18690759 \\
\hline
\end{tabular}


- normal random: $w(v)$ subjects to truncated normal distribution: $w(v) \sim N\left(\mu, \sigma^{2}\right)$, where $w(v) \in(1,+\infty), \mu=100$ and $\sigma=25$.

Therefore, we obtain totally four benchmarks of vertex-weighted graphs, which are denoted by "Repository_200", "Repository_normal", "KONECT_200" and "KONECT_normal”, respectively.

We realize that the way of generating weights may have considerable impact on the performance of algorithms. Indeed, a recent paper has commented on this phenomenon (McCreesh et al., 2017). In particular, McCreesh et al. (2017) mentioned that, when weights are chosen to be between 1 and 200 , the vertex weights would be expected to be more important than degree. So, we also use a normal distribution method to generate weights, for which we would expect degrees become more important. We use these two ways to generate weights, in order to evaluate algorithms with two typical situations.

\subsection{Competitors}

In the following experimental comparison, we compare FastWClq with three state-of-the-art MWCP algorithms, namely WLMC (Jiang et al., 2017), TSM-MWC (Jiang et al., 2018) and SCCWalk4L (Wang et al., 2020).

- WLMC (Jiang et al., 2017) is an exact algorithm for solving the MWCP problem on large graphs. It is the first exact solver that can obtain very good solution values on large graphs, compared with some heuristic solvers. WLMC contains a preprocess procedure to order the vertex and then to reduce the size of graph. It also uses the incremental vertex-weight splitting technique to reduce the number of branches in the search space. The source code of WLMC is available online ${ }^{6}$.

- TSM-MWC (Jiang et al., 2018) is a recent BnB-based exact MWCP algorithm which incorporates a novel two-stage MaxSAT reasoning approach. According to the literature, TSM-MWC is the currently best exact algorithm for MWCP on large instances. As reported by Jiang et al. (2018), TSM-MWC significantly outperforms the WLMC algorithm on a broad range of large real-world graphs. This is the only exact algorithm that shows significantly better performance than the milestone exact MWCP algorithm WLMC on large real-world graphs. The source code of TSM-MWC is available online ${ }^{7}$.

- SCCWalk4L (Wang et al., 2020) is a very recent local search algorithm for MWCP, which shows significantly better performance on large graphs than previous heuristic algorithms. SCCWalk4L integrates powerful local search techniques including configuration checking, walk perturbation as well as BMS. The source code of SCCWalk4L was provided by the authors.

Seen from the literature, WLMC and TSM-MWC establish the latest state of the art in exact algorithms for MWCP on large graphs. As for heuristic algorithms, SCCWalk4L represents the latest state of the art in solving large graphs. It is reported that SCCWalk4L outperforms other recent heuristic algorithms including RRWL (Fan et al., 2017), ReTS1 and ReTS2 (Zhou et al., 2017).

6. http://www.mis.u-picardie.fr/ cli/wlmc2.zip

7. http://home.mis.u-picardie.fr/ cli/tsm-release.zip 


\subsection{Experimental Preliminaries}

FastWClq is implemented in $\mathrm{C}++$, and its source code is available online ${ }^{8}$. Parameters $t_{0}$ and $t_{\max }$ for dynamic BMS heuristic are set to 4 and $64\left(=2^{6}\right)$. Three competitors including WLMC, TSMMWC and SCCWalk4L were implemented in C++ by their authors. For SCCWalk4L, we used the following default values: $\ell=4000$ and $r=50$ (Wang et al., 2020). All algorithms are complied with $\mathrm{g}++$ version 4.7 with the $-\mathrm{O} 3$ optimization flag. FastWClq and SCCWalk4L both are run 10 times on each graph. For the exact algorithms TSM-MWC and WLMC, we run them once on each graph. We test the algorithms with time limit of 100 seconds, 300 seconds and 600 seconds, where the run time of each algorithm includes the time for reading graphs (as some algorithms do non-trivial works including building complex data structure and some initializations during reading the graph). We report the detailed results for the time limit of 100 seconds, while give a summary on all time limits.

The experiments are carried out on a computing cluster consisting of computing nodes equipped with dual 56-core, 2.00GHz Intel Xeon E7-4830 CPUs, 35 MB L3 cache and 256 GB RAM, running Ubuntu version 16.04.5 LTS.

For each graph, we report the largest clique weight ("best") found by each algorithm, the average clique weight over all runs ("avg") and the average run time when algorithm obtains the largest clique weight. If an algorithm fails to provide a solution for an instance, then the corresponding column is marked as "N/A". If an algorithm proves the optimality of its solution, the corresponding column is marked with a “*”.

\subsection{Experimental Results}

In this subsection, we will report the experiment results of FastWClq and the state-of-the-art competitors on large sparse graphs.

\subsubsection{RESUlTS ON REPOSITORY_200}

The results on Repository_200 are presented in Table 3. FastWClq performs better on 29 instances than SCCWalk4L under the same cutoff time (100s). For the remaining instances, FastWClq finds better average solution values than SCCWalk4L, with only three exceptions. Observed from the results, FastWClq computes larger-weight cliques than the two exact solvers TSM-MWC and WLMC for 18 and 11 instances, respectively, while TSM-MWC and WLMC find better cliques for only the two instances soc-flickr-und and web-wikipedia_link. FastWClq computes the optimal solution for 53 instances, more than any other algorithm; although, it only proves the optimality of its solutions in 36 cases. Although they find fewer optimal solutions, TSM-MWC, and WLMC are highly successful at proving optimality, proving optimality in 45 and 52 instances, respectively.

\subsubsection{RESULTS ON REPOSITORY_NORMAL}

The comparison of FastWClq, TSM-MWC, WLMC and SCCWalk4L on Repository_normal is given in Table 4. Once again, FastWClq has the best performance. Firstly, FastWClq finds cliques of larger weight than SCCWalk4L for 32 graphs, while FastWClq finds a worse solution than SCCWalk4L for only one graph. FastWClq finds solutions of the same or higher quality than the two exact solvers TSM-MWC and WLMC on all but one instance (instance soc-flickr-und), and on 46

8. http://lcs.ios.ac.cn/ caisw/CLQ.html 
Table 3. Experiment results of FastWClq and the state-of-the-art competitors on Repository_200 benchmark.

\begin{tabular}{|c|c|c|c|c|c|c|c|c|}
\hline \multirow{2}{*}{ Instance } & \multicolumn{2}{|l|}{ FastWClq } & \multicolumn{2}{|l|}{ TSM-MWC } & \multicolumn{2}{|l|}{ WLMC } & \multicolumn{2}{|l|}{ SCCWalk4L } \\
\hline & size & time & size & time & size & time & size & time \\
\hline bn-human...1-bg & 20184 (19678) & 31.08 & 19547 (19547) & 89.88 & 19185 (19185) & 81.88 & $14506(13568.6)$ & 99.53 \\
\hline bn-human...2-bg & 19189 (19189) & 16.54 & 16396 (16396) & 70.58 & $16102(16102)$ & 99.19 & $11804(9478.8)$ & 99.51 \\
\hline ca-coauthors-dblp & $37884 *(37884)$ & 5.07 & $37884 *(37884)$ & 13.09 & $37884 *(37884)$ & 13.09 & $37884 *(37884)$ & 26.88 \\
\hline ca-dblp-2012 & $14108 *(14108)$ & 0.96 & $14108 *(14108)$ & 1.29 & $14108 *(14108)$ & 1.39 & $14108 *(14108)$ & 2.11 \\
\hline ca-hollywood-2009 & $222720 *(222720)$ & 55.18 & $222720 *(222720)$ & 67.36 & $222720 *(222720)$ & 69.48 & $15367(11009.9)$ & 99.61 \\
\hline channel-500x $100 \times 100-b 050$ & $796 *(796)$ & 5.72 & $796 *(796)$ & 43.78 & $796 *(796)$ & 41.89 & 796 (796) & 69.47 \\
\hline dbpedia-link & $4156(2507.2)$ & 93.54 & N/A (N/A) & N/A & N/A (N/A) & N/A & N/A (N/A) & N/A \\
\hline delaunay_n22 & $796 *(796)$ & 5.58 & $796 *(\mathbf{7 9 6})$ & 15.22 & $796 *(\mathbf{7 9 6})$ & 14.72 & $796 *(750.3)$ & 47.86 \\
\hline delaunay_n23 & $798 *(\mathbf{7 9 8})$ & 12.78 & $798 *(\mathbf{7 9 8})$ & 29.99 & $798 *(\mathbf{7 9 8})$ & 29.68 & $798 *(672)$ & 80.99 \\
\hline delaunay_n24 & $797 *(797)$ & 25.36 & N/A (N/A) & N/A & $797 *(797)$ & 65.73 & $566(465.9)$ & 97.28 \\
\hline friendster & 5511* (4982.4) & 86.42 & $5511 *(5511)$ & 79.09 & $5511 *(5511)$ & 81.97 & $567(500)$ & 99.91 \\
\hline hugebubbles- 00020 & $400 *(400)$ & 49.83 & N/A (N/A) & N/A & $400 *(400)$ & 60.6 & $399(384.8)$ & 92.18 \\
\hline hugetrace- 00010 & $400 *(400)$ & 19.5 & N/A (N/A) & N/A & $400 *(400)$ & 28.19 & 399 (399) & 56.15 \\
\hline hugetrace- 00020 & $400 *(400)$ & 29.17 & N/A (N/A) & N/A & $400 *(400)$ & 43.19 & $400(398.9)$ & 79.99 \\
\hline inf-europe_osm & $646 *(646)$ & 55.99 & N/A (N/A) & N/A & $646 *(646)$ & 85.98 & 267 (267) & 91.65 \\
\hline inf-germany_osm & $597 *(597)$ & 14.3 & N/A (N/A) & N/A & $597 *(597)$ & 19.89 & $\mathbf{5 9 7} *(524.6)$ & 63.96 \\
\hline inf-road-usa & $766 *(766)$ & 29.89 & N/A (N/A) & N/A & $766 *(766)$ & 51.19 & $\mathbf{7 6 6} *(537.4)$ & 88.23 \\
\hline inf-roadNet-CA & $752 *(752)$ & 1.56 & $752 *(752)$ & 4.19 & $752 *(752)$ & 4.38 & $752 *(752)$ & 6.98 \\
\hline inf-roa & $669 *(669)$ & 0.92 & $669 *(669)$ & 2.18 & $669 *(669)$ & 2.39 & 669 (669) & 3.12 \\
\hline rec-dating & 1699 (1524.2) & 40.39 & $1699 *(\mathbf{1 6 9 9})$ & 24.59 & 1699*(1699) & 25.14 & 1699 (1699) & 24.96 \\
\hline rec-epinions & 1054 (998.4) & 42.36 & $1054 *(\mathbf{1 0 5 4})$ & 73.79 & $1054 *(\mathbf{1 0 5 4})$ & 76.12 & 1054 (1054) & 50.33 \\
\hline rec-libimseti-dir & $1938(1781.2)$ & 44.83 & $1938 *(\mathbf{1 9 3 8})$ & 29.16 & $1938 *(\mathbf{1 9 3 8})$ & 28.90 & 1938 (1938) & 27.94 \\
\hline rgg_n_2_23_s0 & $2407 *(2407)$ & 27.18 & $2407 *(2407)$ & 65.94 & $2407 *(2407)$ & 64.92 & $1103(1042.67)$ & 97.21 \\
\hline rgg_n_2_24_s0 & $2514 *(2514)$ & 59.37 & N/A (N/A) & N/A & N/A (N/A) & N/A & N/A (N/A) & N/A \\
\hline rt-retweet-crawl & $1367 *(1367)$ & 4.47 & $1367 *(1367)$ & 3.63 & $1367 *(1367)$ & 3.85 & $1367(1367)$ & 19.4 \\
\hline sc-ldoor & $4081 *(\mathbf{4 0 8 1})$ & 3.91 & $4081 *(\mathbf{4 0 8 1})$ & 17.39 & $4081 *(\mathbf{4 0 8 1})$ & 17.48 & $4081(4054.4)$ & 60.65 \\
\hline sc-msdoor & $4088 *(\mathbf{4 0 8 8})$ & 1.46 & $4088 *(\mathbf{4 0 8 8})$ & 7.49 & $4088 *(\mathbf{4 0 8 8})$ & 7.49 & 4088 (4086.6) & 29.51 \\
\hline sc-pwtk & $4620 *(4620)$ & 0.49 & $4620 *(4620)$ & 4.34 & $4620 *(4620)$ & 4.48 & $4620(4620)$ & 8.28 \\
\hline sc-rel9 & $572 *(\mathbf{5 7 2})$ & 34.39 & $572 *(\mathbf{5 7 2})$ & 29.14 & $572 *(\mathbf{5 7 2})$ & 28.79 & $572(417.2)$ & 62.06 \\
\hline sc-shipsec1 & $3540 *(3540)$ & 0.23 & $3540 *(3540)$ & 1.21 & $3540 *(3540)$ & 1.48 & $3540 *$ & 2.57 \\
\hline sc-shipsec5 & $4524 *(4524)$ & 0.18 & $4524 *(4524)$ & 1.74 & $4524 *(4524)$ & 1.91 & $4524 *(4$ & 3.36 \\
\hline soc-FourSquare & 3064 (3064) & 50.64 & $3064 *(3064)$ & 12.08 & 3064* (3064) & 12.07 & $2025(18$ & 93.66 \\
\hline soc-LiveMocha & 1784 (1784) & 11.71 & $1784 *(1784)$ & 2.58 & $1784 *(1784)$ & 2.63 & 1784 (1784) & 7.88 \\
\hline soc-buzznet & 2981 (2981) & 33.14 & $2981 *(\mathbf{2 9 8 1})$ & 8.4 & $2981 *(\mathbf{2 9 8 1})$ & 8.59 & 2981 (2977) & 56.51 \\
\hline soc-delicious & 1547 (1547) & 1.03 & $1547 *(\mathbf{1 5 4 7})$ & 1.25 & $1547 *(\mathbf{1 5 4 7})$ & 1.48 & $1547(1$ & 34 \\
\hline soc-digg & $5303(5302.6)$ & 48.5 & $5303 *(\mathbf{5 3 0 3})$ & 14.41 & $5303 *(\mathbf{5 3 0 3})$ & 14.98 & $5303(50$ & 58.17 \\
\hline soc-dogster & $4418(4400.8)$ & 65.82 & $4418 *(\mathbf{4 4 1 8})$ & 10.16 & $4418 *(4$ & 9.86 & $4418(4$ & 87.2 \\
\hline soc-flickr & 7083 (7083) & 40.22 & $7083 *(7083)$ & 4.08 & 7083* (7083) & 4.55 & $7083(7$ & 18.53 \\
\hline soc-flickr-und & $10126(9860.6)$ & 32.88 & $10127 *(10127)$ & 44.94 & 9329 (9329) & 89.83 & 6906 (5309) & 99.35 \\
\hline soc-flixster & 3805 (3805) & 10.29 & $3805 *(3805)$ & 10.87 & & 10.93 & $3805(3$ & 32.43 \\
\hline soc- -1 & 17 & 10.62 & $1773 *(1$ & 6.26 & 177 & 5.72 & 1773 & 14.51 \\
\hline soc-livejournal & $21368 *(21368)$ & 43.02 & $21368 *(21368)$ & 39.31 & $21368 *(21368)$ & 39.27 & $9183(3009.6)$ & 95.03 \\
\hline soc-livejournal-user-groups & $957(860.4)$ & 76.83 & N/A (N/A) & N/A & N/A (N/A) & N/A & N/A (N/A) & N/A \\
\hline soc-ljournal-2008 & $40432(40432)$ & 57.37 & $40432 *(40432)$ & 68.06 & $40432 *(40432)$ & 70.63 & $2709(1649.5)$ & 95.48 \\
\hline soc-orkut & $4439(3628.8)$ & 91.29 & N/A (N/A) & N/A & N/A (N/A) & N/A & N/A (N/A) & N/A \\
\hline soc-orkut-dir & $4262(3656.8)$ & 90.43 & N/A (N/A) & N/A & N/A (N/A) & N/A & N/A (N/A) & N/A \\
\hline soc-pokec & $3191 *(3191)$ & 37.64 & $3191 *(3191)$ & 33.42 & 3191* (3191) & 31.85 & 2994 (1990.9) & 86.46 \\
\hline soc-sinaweibo & N/A (N/A) & N/A & N/A (N/A) & N/A & N/A (N/A) & N/A & N/A (N/A) & N/A \\
\hline tter-higgs & $8039 *(8039)$ & 13.31 & $8039 *(8039)$ & 7.98 & $8039 *(8039)$ & 17.88 & $4727(4699.7)$ & 68.63 \\
\hline soc-youtube & $1961 *(1961)$ & 2.49 & $1961 *(1961)$ & 2.39 & $1961 *(1961)$ & 2.88 & $1961(1961)$ & 8.13 \\
\hline soc-youtube-snap & $1787 *(1787)$ & 4.71 & $1787 *(1787)$ & 4.78 & $1787 *(1$ & 4.88 & 1787 (1787) & 13.87 \\
\hline & $2872 *(2872)$ & 36.84 & $2872 *(2872)$ & 3427 & $2872 *(2872)$ & 34.42 & 2295 (1992.3) & 75.76 \\
\hline socfb-B-anon & $2662(2662)$ & 41.77 & $2662 *(2662)$ & 30.48 & $2662 *(2662)$ & 29.88 & $2513(2075.4)$ & 81.32 \\
\hline socfb-uci-uni & 453 (331.6) & 93.28 & N/A (N/A) & N/A & N/A (N/A) & N/A & N/A (N/A) & N/A \\
\hline -as-skitter & $5703(\mathbf{5 7 0 3})$ & 9.42 & $5703 *(\mathbf{5 7 0 3})$ & 22.55 & $5703 *(\mathbf{5 7 0 3})$ & 22.89 & $5703(5666.8)$ & 78.48 \\
\hline tech-ip & $668(585)$ & 48.4 & N/A (N/A) & N/A & N/A (N/A) & N/A & 249 (162) & 44.21 \\
\hline twitter_n & 6510 & 91.25 & N/A (N/A) & N/A & N/A (N/A) & N/A & N/A (N/A) & N/A \\
\hline 005 & $10558 *(10558)$ & 0.16 & $10558 *(10558)$ & 1.49 & $10558 *(10558)$ & 1.28 & $10558 *(10558)$ & 2.17 \\
\hline web-baidu-baike & $3814 *(3814)$ & 22.46 & $3814 *(3814)$ & 25.89 & 3814* (3814) & 26.09 & $1743(1656.2)$ & 81.93 \\
\hline web-it-2004 & $45477 *(45477$ & 1.28 & $45477 *(45477)$ & 5.89 & $45477 *(45477)$ & 5.99 & $45477 *(45477)$ & 15.68 \\
\hline web-uk-2005 & $54850 *(54850)$ & 1.55 & $54850 *(54850)$ & 9.28 & $54850 *(54850)$ & 9.39 & $54850 *(54850)$ & 15.08 \\
\hline web-wikipedia-growt & $4741(4741)$ & 54.21 & $4741 *(4741)$ & 63.98 & $4741 *(4741)$ & 64.08 & $1714(1380.3)$ & 97.7 \\
\hline web-wikipedia2009 & $3891 *(3891)$ & 8.68 & $3891 *(3891)$ & 7.38 & $3891 *(3891)$ & 7.59 & 3891 (3891) & 15.18 \\
\hline web-wikipedia_link & $51331(25964.8)$ & 76.25 & 89544 (89544) & 99.78 & 89545 (89545) & 99.99 & N/A (N/A) & N/A \\
\hline wikipedia_link_en & $4624(4618.1)$ & 75.55 & N/A (N/A) & N/A & $4624 *(4624)$ & 68.78 & $1002(707)$ & 93.85 \\
\hline
\end{tabular}


instances it finds these solutions faster. Focusing on the ability of proving optimality, FastWClq, TSM-MWC and WLMC prove the optimality of 36, 45 and 52 instances, respectively. The total number of instances provably solved to optimality by the exact algorithms TSM-MWC and WLMC is more than FastWClq for 17 instances. FastWClq proves the optimality of instance rgg_n_2_24_s0, while the two exact algorithms fail to find any solution within the time limit.

\subsubsection{RESULTS ON KONECT_200}

The experimental results are presented in Table 5, which shows that FastWClq is clearly the best solver on the KONECT_200 benchmark. FastWClq finds the largest-weight cliques for all instances in KONECT_200 except for the two instances flickr-link and libimseti. Furthermore, FastWClq finds larger-weight cliques than TSM-MWC, WLMC and SCCWalk4L for 31, 32, and 38 instances, respectively. For instances where FastWClq and a corresponding competitor obtain the same solution quality (i.e., the same maximum and same average solution values), FastWClq is faster than the other three algorithms, except for six instances. FastWClq proves the optimality of nine solutions that neither TSM-MWC and WLMC prove to be optimal, but FastWClq fails to prove optimality on 16 instances where TSM-MWC and WLMC both succeed in proving optimality.

Table 5. Experiment results of FastWClq and the state-of-the-art competitors on KONECT_200 benchmark.

\begin{tabular}{|c|c|c|c|c|c|c|c|c|}
\hline \multirow{2}{*}{ Instance } & \multicolumn{2}{|l|}{ FastWClq } & \multicolumn{2}{|l|}{ TSM-MWC } & \multicolumn{2}{|l|}{ WLMC } & \multicolumn{2}{|c|}{ SCCWalk4L } \\
\hline & size & time & size & time & size & time & size & time \\
\hline actor-collaboration & $25500 *(25500)$ & 9.54 & $25500 *(25500)$ & 25.99 & $25500^{*}(25500)$ & 25.55 & $25500(25500)$ & 76.5 \\
\hline amazon0601 & $2127 *(2127)$ & 1.6 & $2127 *(2127)$ & 2.79 & $2127 *(2127)$ & 2.96 & $2127 *(2127)$ & 3.9 \\
\hline as-skitter & $5703(5703)$ & 8.32 & $5703 *(5703)$ & 22.79 & $5703 *(5703)$ & 22.49 & $5703(5703)$ & 73.96 \\
\hline citeseer & $1679 *(1679)$ & 1.72 & $1679 *(1679)$ & 2.29 & $1679 *(1679)$ & 2.38 & 1679 (1679) & 5.34 \\
\hline com-amazon & $1301 *(1301)$ & 0.51 & $1301 *(1301)$ & 1.18 & $1301 *(1301)$ & 1.46 & $1301 *(1301)$ & 1.38 \\
\hline com-dblp & $10668 *(10668)$ & 0.32 & 10668*(10668) & 1.26 & $10668^{*}(10668)$ & 1.39 & $10668 *(10668)$ & 1.48 \\
\hline com-youtube & $1967 *(1967)$ & 5.6 & $1967 *(1967)$ & 4.28 & $1967 *(1967)$ & 4.88 & 1967 (1967) & 11.3 \\
\hline dbpedia-all & $2547 *(2547)$ & 15.95 & N/A (N/A) & N/A & N/A (N/A) & N/A & 2547* (1196) & 97.25 \\
\hline dbpedia-link & $1258(701.111)$ & 95.1 & N/A (N/A) & N/A & N/A (N/A) & N/A & N/A (N/A) & N/A \\
\hline digg-friends & 3668 (3666.5) & 43.72 & N/A (N/A) & N/A & N/A (N/A) & N/A & $203(141.2)$ & 12.15 \\
\hline douban & $1197 *(1197)$ & 0.06 & $1197 *(1197)$ & 0.01 & $1197 *(1197)$ & 0.71 & 1197 (1197) & 0.73 \\
\hline elec & $1936(1936)$ & 0.14 & $1936 *(1936)$ & 2.79 & $1936 *(1936)$ & 2.88 & $1936(1936)$ & 7.32 \\
\hline email-EuAll & $2082 *(2082)$ & 0.19 & $2082 *(2082)$ & 0.79 & $2082 *(2082)$ & 0.99 & $2082(2082)$ & 0.81 \\
\hline enron & $3496(3496)$ & 0.24 & $3496 *(3496)$ & 18.59 & $3496 *(3496)$ & 18.75 & $3496(3496)$ & 16.41 \\
\hline epinions & 10037 (10037) & 0.14 & $10037 *(10037)$ & 0.98 & $10037^{*}(10037)$ & 1.08 & 10037 (10037) & 6.88 \\
\hline facebook-wosn-links & $3618(3618)$ & 0.35 & $3618 *(3618)$ & 42.18 & $3618 *(3618)$ & 42.31 & $3618(3618)$ & 27.5 \\
\hline facebook-wosn-wall & $1175 *(1175)$ & 0.35 & N/A (N/A) & N/A & N/A (N/A) & N/A & $526(232.1)$ & 49 \\
\hline flickr-growth & $11508(11268.7)$ & 36.04 & 10917 (10917) & 97.25 & 10678 (10678) & 96.47 & 7924 (5498.4) & 85.11 \\
\hline flickr-links & 10027 (9904) & 47.29 & $10036 *(10036)$ & 44.09 & $9242(9242)$ & 94.46 & 9225 (7940.7) & 99.86 \\
\hline flickrEdges & $60280 *(60280)$ & 1.07 & $60280 *(60280)$ & 2.48 & $60280 *(60280)$ & 2.58 & $60280 *(60280)$ & 14.7 \\
\hline flixster & $3399(3399)$ & 10.29 & $3399 *(3399)$ & 10.29 & $3399 *(3399)$ & 10.27 & $3399(3399)$ & 26.39 \\
\hline hyves & $1953 *(1953)$ & 2.5 & $1953 *(1953)$ & 4.67 & $1953 *(1953)$ & 4.88 & $1953(1953)$ & 7.76 \\
\hline lasagne-yahoo & $457 *(457)$ & 10.76 & $457 *(457)$ & 32.5 & $457 *(457)$ & 31.83 & 444 (431.9) & 52.35 \\
\hline libimseti & $1092(999)$ & 60.69 & $1099 *(1099)$ & 94.78 & $1099 *(1099)$ & 95.47 & $1099(1099)$ & 31.22 \\
\hline link-dynamic-dewiki & $11669(11669)$ & 70.89 & N/A (N/A) & N/A & N/A (N/A) & N/A & $178(102.556)$ & 94.81 \\
\hline link-dynamic-frwiki & $8637 *(8637)$ & 66.24 & N/A (N/A) & N/A & N/A (N/A) & N/A & $662(174)$ & 71.39 \\
\hline link-dynamic-itwiki & $5776(5776)$ & 25.58 & N/A (N/A) & N/A & N/A (N/A) & N/A & $538(203.4)$ & 43.15 \\
\hline link-dynamic-nlwiki & $5118 *(5118)$ & 15.18 & N/A (N/A) & N/A & N/A (N/A) & N/A & $199(126.4)$ & 28.93 \\
\hline link-dynamic-plwiki & $5202(5202)$ & 52.07 & N/A (N/A) & N/A & N/A (N/A) & N/A & $191(135.5)$ & 30.78 \\
\hline link-dynamic-simplewiki & $3988 *(3988)$ & 0.48 & $3988 *(3988)$ & 23.44 & $3988 *(3988)$ & 23.7 & $3988(3988)$ & 19.79 \\
\hline
\end{tabular}


Table 5. (Continued) Experiment results of FastWClq and the state-of-the-art competitors on KONECT_200 benchmark.

\begin{tabular}{|c|c|c|c|c|c|c|c|c|}
\hline \multirow{2}{*}{ Instance } & \multicolumn{2}{|l|}{ FastWClq } & \multicolumn{2}{|l|}{ TSM-MWC } & \multicolumn{2}{|l|}{ WLMC } & \multicolumn{2}{|l|}{ SCCWalk4L } \\
\hline & size & time & size & time & size & time & size & time \\
\hline livejournal-links & $38550(38550)$ & 66.03 & $38550 *(38550)$ & 71.09 & $38550 *(38550)$ & 69.23 & $5106(2126)$ & 96.6 \\
\hline livemocha & $1784(1784)$ & 10.63 & $1784 *(1784)$ & 2.24 & $1784 *(1784)$ & 2.9 & $1784(1784)$ & 7.04 \\
\hline lkml-reply & $4763(4763)$ & 0.59 & N/A (N/A) & N/A & N/A (N/A) & N/A & $202(191.5)$ & 41.54 \\
\hline loc-gowalla_edges & $3053 *(\mathbf{3 0 5 3})$ & 0.24 & $3053 *(\mathbf{3 0 5 3})$ & 1 & $3053 *(\mathbf{3 0 5 3})$ & 1.19 & $3053(2834.6)$ & 20.51 \\
\hline munmun_digg & $686 *(686)$ & 0.02 & $686 *(686)$ & 2.9 & $686^{*}(686)$ & 3.08 & $686(686)$ & 6.92 \\
\hline munmun_twitter_social & $750 *(750)$ & 0.4 & $750 *(750)$ & 1.12 & $750 *(750)$ & 1.3 & $750(750)$ & 1.95 \\
\hline orkut-links & $5290(4429.4)$ & 90.24 & N/A (N/A) & N/A & N/A (N/A) & N/A & N/A (N/A) & N/A \\
\hline patentcite & $1438 *(1438)$ & 32.47 & $1438 *(1438)$ & 27.99 & $1438 *(1438)$ & 28.19 & $1355(1006.8)$ & 79.43 \\
\hline petster-carnivore & $119297 *(119297)$ & 11.79 & $119188(119188)$ & 98.38 & $119210(119210)$ & 99.39 & $5251(4802.7)$ & 95.82 \\
\hline petster-friendships-cat-uniq & $9364(9293.8)$ & 40.05 & $9364 *(9364)$ & 45.79 & $9364 *(9364)$ & 52.09 & $1962(18$ & 93.33 \\
\hline petster-friendships-do & $4418(4411.8)$ & 75.91 & $4418 *(4418)$ & 22.58 & $4418 *(4418)$ & 21.99 & $4418(4404.2)$ & 77.56 \\
\hline roadNet-CA & $790 *(790)$ & 1.46 & $790 *(790)$ & 4.09 & $790 *(790)$ & 4.19 & $790 *(790)$ & 5.88 \\
\hline roadNet-PA & $774 *(774)$ & 0.71 & $774 *(774)$ & 2.09 & $774 *(774)$ & 2.2 & $774 *(774)$ & 2.77 \\
\hline roadNet-TX & $772 *(772)$ & 0.9 & $772 *(772)$ & 2.79 & $772 *(772)$ & 2.58 & $772 *(772)$ & 3.41 \\
\hline slashdot-thre & $720 *(720)$ & 0.06 & $720 *(720)$ & 3.19 & & 3.39 & $720(720)$ & 4.87 \\
\hline soc-LiveJ & $31814(31814)$ & 58.17 & $14 *(31$ & 68.18 & $4 *(31)$ & 63.99 & $5881(290$ & 95.94 \\
\hline soc-pokec-r & $2993 *(2$ & & $2993 *(29$ & 34.09 & & 34.19 & $2584(1$ & 75.2 \\
\hline trec-wt10g & 918 & 1.99 & $*(91$ & & $(9180)$ & 8.49 & $0(9$ & 14.78 \\
\hline & & 25 & $*(2$ & 25.49 & 9) & 26.78 & 079) & 38.13 \\
\hline & & 25 & $=(50$ & 4.79 & 5 & 4.74 & $4 *($ & 6.86 \\
\hline wel & 9 & 0.27 & $3 *(1$ & 1.19 & 133) & 1.4 & $19133^{*}$ & 2.47 \\
\hline web- & $(7)$ & 0.48 & $3 *(77$ & 4.69 & (7753) & 4.75 & $7753(7$ & 16.65 \\
\hline wiki-' & 2985 (2985) & 10.37 & $2985 *(2985)$ & 15.09 & $*(2985)$ & 15.09 & 2985 (2985) & 21.62 \\
\hline wiki_talk_ar & $3292 *(3292)$ & 1.74 & N/A (N/A) & N/A & N/A (N/A) & N/A & 354 (172) & 3.65 \\
\hline wiki_t & $4391(4390)$ & 33.56 & N/A (N/A) & N/A & & 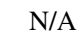 & 21 & 8.3 \\
\hline wiki_ & .3) & 72.65 & N/A) & N/A & (A) & N/A & $3371(2$ & 99.71 \\
\hline wiki_talk_es & $3831(3831)$ & 2.29 & N/A (N/A) & & & 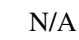 & $594(188$ & 3.24 \\
\hline wiki_talk_fr & 141 & 4.25 & N/A (N/A) & & & & 17 & 7.22 \\
\hline wiki_ & 4 & 3. & & & & & & 4.5 \\
\hline & & & & & & & & 5.34 \\
\hline wil & & 1.76 & (A) & A & & I/A & & 3.65 \\
\hline & 3 & 1.69 & (A) & A & & I/A & 2 & 8.13 \\
\hline wik & 97) & 2.02 & N/A) & N/A & /A) & N/A & 136) & 16.39 \\
\hline wiki & $4741(4741)$ & 48.08 & N/A (N/A) & N/A & /A) & N/A & $1927(1784.2)$ & 98.07 \\
\hline wikipedia_link_de & $83708 *(76772.2)$ & 89.54 & N/A (N/A) & N/A & N/A) & N/A & $1382(860)$ & 99.46 \\
\hline wikipedia_link_en & N/A (N/A) & N/A & N/A (N/A) & N/A & N/A (N/A) & N/A & N/A (N/A) & N/A \\
\hline wikipedia_link_fr & 106234 (37249.6) & 96.1 & N/A (N/A) & N/A & N/A (N/A) & $\mathrm{N}$ & N/A (N/A) & N/A \\
\hline dia_link_it & $87969 *(87969)$ & 71.33 & N/A (N/A) & N/A & N/A (N/A) & $\mathrm{N}$ & $2097(1152.5)$ & 98.28 \\
\hline a_link_ja & $89015 *(890$ & 73.69 & N/A (N/A) & N/A & N/A (N/A) & $\mathrm{N}$ & 2447 (1769.4) & 97.33 \\
\hline wikip & 8299 & 42.08 & N/A (N/A) & N/A & & $\mathrm{N}$ & $1946(17$ & 97.76 \\
\hline & 1036 & 40.46 & & & & & & 86.07 \\
\hline wil & & 71.78 & & & & & & 98.98 \\
\hline wikis & $2 *(11$ & 0.21 & $1192 *(1192$ & 3.39 & $2 *(1192)$ & 4.08 & $1192(1$ & 14.15 \\
\hline & $5037 *(5037)$ & 0.21 & $5037 *(5037)$ & 0.78 & $5037 *(5037)$ & 0.79 & $5037 *(5$ & 0.94 \\
\hline youtube-links & 1837 (1837) & 4.13 & $1837 *(1837)$ & 4.49 & $1837 *(1837)$ & 4.59 & 1837 (1837) & 10.37 \\
\hline youtube-u-growth & $2321(2321)$ & 17.68 & $2321 *(2321)$ & 27.89 & $2321 *(2321)$ & 27.09 & $2321(2321)$ & 55.32 \\
\hline zhishi-baidu-internallink & $3814 *(3814)$ & 21 & $3814 *(3814)$ & 48.09 & $3814 *(3814)$ & 46.98 & $1823(1624.6)$ & 64.88 \\
\hline zhishi-baidu-relatedpages & $8938(8938)$ & 1.14 & $8938 *(8938)$ & 49.78 & $8938 *(8938)$ & 49.8 & 8938 (8938) & 37.7 \\
\hline & $26110 *(2611$ & 20.25 & $26110 *(26110)$ & 33.19 & $26110 *(26110)$ & 32.79 & $26110(26110)$ & 81.06 \\
\hline zhishi-hudong-relatedpages & $2262 *(\mathbf{2 2 6 2})$ & 28.38 & $2262 *(\mathbf{2 2 6 2})$ & 50.19 & $2262 *(\mathbf{2 2 6 2})$ & 49.97 & $2262(1710.1)$ & 81.73 \\
\hline
\end{tabular}


Table 4. Experiment results of FastWClq and the state-of-the-art competitors on Repository_normal benchmark.

\begin{tabular}{|c|c|c|c|c|c|c|c|c|}
\hline \multirow{2}{*}{ Instance } & \multicolumn{2}{|l|}{ FastWClq } & \multicolumn{2}{|l|}{ TSM-MWC } & \multicolumn{2}{|l|}{ WLMC } & \multicolumn{2}{|l|}{ SCCWalk4L } \\
\hline & size & time & size & time & size & time & size & time \\
\hline bn-human...1-bg & $19840(19395.9)$ & 41.78 & $17886(17886)$ & 89.03 & $17792(17792)$ & 83.39 & $15556(12576.9)$ & 99.04 \\
\hline bn-human...2-bg & 19981 (19981) & 15.8 & $15761(15761)$ & 74.44 & $15761(15761)$ & 94.46 & $12268(9862.4)$ & 99.52 \\
\hline ca-coauthors-dblp & $33511 *(33511)$ & 4.87 & $33511 *(33511)$ & 12.92 & $33511 *(33511)$ & 12.94 & $33511 *(33511)$ & 29.74 \\
\hline ca-dblp-2012 & $11761 *(11761)$ & 0.9 & $11761^{*}(11761)$ & 1.28 & $11761 *(11761)$ & 1.25 & $11761 *(11761)$ & 2.26 \\
\hline ca-hollywood-2009 & $221751 *(221751)$ & 54.05 & $221751 *(221751)$ & 67.31 & $221751 *(221751)$ & 68.95 & 43588 (17032) & 96.95 \\
\hline channel-500 $\times 100 \times 100-b 050$ & $683 *(683)$ & 18.54 & $683 *(683)$ & 45.89 & $683 *(683)$ & 46.29 & 654 (601.7) & 85.36 \\
\hline dbpedia-link & $2971(2478.1)$ & 92.99 & N/A (N/A) & N/A & N/A (N/A) & N/A & N/A (N/A) & N/A \\
\hline delaunay_n22 & $612 *(612)$ & 5.22 & $612 *(\mathbf{6 1 2})$ & 15.19 & $612 *(612)$ & 15.11 & $612 *(486.6)$ & 69.33 \\
\hline delaunay_n23 & $610 *(610)$ & 11.33 & $610 *(610)$ & 30.39 & $610 *(610)$ & 31.06 & $491(454.3)$ & 72.54 \\
\hline delaunay_n24 & $612 *(612)$ & 22.47 & N/A (N/A) & N/A & $612 *(612)$ & 63.58 & $448(413.889)$ & 91.03 \\
\hline friendster & 3886 (3886) & 84.21 & $3886 *(3886)$ & 77.84 & $3886 *(3886)$ & 77.98 & 794 (493) & 98.84 \\
\hline hugebubbles- 00020 & $434 *(434)$ & 52.86 & N/A (N/A) & N/A & $434 *(434)$ & 61.48 & $316(290.3)$ & 82.02 \\
\hline hugetrace- 00010 & $434 *(434)$ & 22.26 & N/A (N/A) & N/A & $434 *(434)$ & 30.59 & $434(344.8)$ & 60.92 \\
\hline hugetrace- 00020 & $410 *(410)$ & 33.51 & N/A (N/A) & N/A & $410 *(410)$ & 42.99 & $317(301)$ & 75.88 \\
\hline inf-europe_osm & $515 *(515)$ & 61.91 & N/A (N/A) & N/A & $515 *(515)$ & 90.69 & $263(201.2)$ & 98.41 \\
\hline inf-germany_osm & $447 *(447)$ & 14.48 & N/A (N/A) & N/A & $447 *(447)$ & 20.19 & $447 *(447)$ & 44.81 \\
\hline inf-road-usa & $521 *(521)$ & 31.59 & N/A (N/A) & N/A & $521 *(521)$ & 53.99 & $\mathbf{5 2 1} *(351.9)$ & 90.63 \\
\hline inf-roadNet-CA & $507 *(507)$ & 1.72 & $507 *(507)$ & 4.29 & $507 *(507)$ & 4.29 & 507 & 6.22 \\
\hline inf-roadNet-PA & $507 *(507)$ & 0.86 & $507 *(507)$ & 2.23 & $507 *(507)$ & 2.49 & $507 *(507)$ & 3.25 \\
\hline rec-dating & $1305(1203.1)$ & 37.59 & $1305 *(\mathbf{1 3 0 5})$ & 25.45 & $1305^{*}(\mathbf{1 3 0 5})$ & 26.37 & 1305 & 27.12 \\
\hline rec-epinions & 923 (889.4) & 55.6 & $947 *(947)$ & 71.73 & $947 *(947)$ & 75.67 & 947 & 45.18 \\
\hline rec-libimseti-dir & $1465(1409.2)$ & 28.52 & $1465 *(\mathbf{1 4 6 5})$ & 27.79 & $1465 *(\mathbf{1 4 6 5})$ & 27.77 & 1465 (1465) & 30.27 \\
\hline rgg_n_2_23_s0 & $2245 *(2245)$ & 25.8 & $2245 *(2245)$ & 62.89 & $2245 *$ & 64.76 & 1194 & 99.3 \\
\hline rgg_n_2_24_s0 & $2359 *(2359)$ & 58.1 & N/A (N/A) & N/A & N/A (N/A) & N/A & N/A (N/A) & N/A \\
\hline rt-retweet-crawl & $1477 *(\mathbf{1 4 7 7})$ & 4.41 & $1477 *(\mathbf{1 4 7 7})$ & 3.74 & $1477 *(\mathbf{1 4 7 7})$ & 3.67 & $1477(1411.5)$ & 26.43 \\
\hline sc-ldoor & $2589 *(2589)$ & 5.45 & $2589 *(2589)$ & 18.55 & $2589 *(2589)$ & 18.68 & 2554 & 67.93 \\
\hline sc-msdoor & $2592 *(2592)$ & 2.21 & $2592 *(2592)$ & 8.28 & $2592 *(2592)$ & 8.57 & $2591(2528.1)$ & 41.77 \\
\hline sc-pwtk & $2876^{*}(\mathbf{2 8 7 6})$ & 0.74 & $2876 *(2876)$ & 4.79 & $2876 *(2876)$ & 5.01 & $2876(2831.1)$ & 53.78 \\
\hline sc-rel9 & $441 *(441)$ & 39.1 & $441 *(441)$ & 28.74 & $441 *(441)$ & 28.44 & $378(359.1)$ & 78.15 \\
\hline sc-shipsec1 & $2514 *(2514)$ & 0.18 & $2514 *(2514)$ & 1.14 & $2514 *(2514)$ & 1.21 & 2514 (2514) & 4.42 \\
\hline sc-shipsec5 & $2754 *(2754)$ & 0.31 & $2754 *(2754)$ & 1.93 & $2754 *(2754)$ & 2.09 & 2754 & 4.37 \\
\hline soc-FourSquare & 3198 (3198) & 17.54 & $3198 *(3198)$ & 12.19 & $3198 *(3198)$ & 11.84 & $2008(1955.2)$ & 95.76 \\
\hline soc-LiveMocha & 1599 (1599) & 7.21 & $1599 *(1599)$ & 2.36 & $1599 *(1599)$ & 2.61 & 1599 (1599) & 8.18 \\
\hline soc-buzznet & 2944 (2943) & 65.3 & $2944 *(\mathbf{2 9 4 4})$ & 6.33 & $2944 *(2944)$ & 6.48 & 2944 (2944) & 36.85 \\
\hline soc-delicious & $2158(2158)$ & 0.87 & $2158 *(2158)$ & 1.25 & $2158 *(2158)$ & 1.25 & 2158 & 11.82 \\
\hline soc-digg & $5036(\mathbf{5 0 3 6})$ & 12.38 & $5036 *(\mathbf{5 0 3 6})$ & 15.02 & $5036 *(\mathbf{5 0 3 6})$ & 14.48 & 5036 & 63.64 \\
\hline soc-dogster & $4581(4574.1)$ & 91.23 & $4581 *(\mathbf{4 5 8 1})$ & 10.18 & $4581 *(\mathbf{4 5 8 1})$ & 9.82 & $4581(4579.4)$ & 83.73 \\
\hline soc-flickr & $5930(5927.9)$ & 14.97 & $5930 *(\mathbf{5 9 3 0})$ & 4.13 & $5930 *(\mathbf{5 9 3 0})$ & 4.48 & $5930(\mathbf{5 9 3 0})$ & 19.77 \\
\hline soc-flickr-und & 9856 (9712.5) & 33.85 & $9929 *(9929)$ & 75.5 & 9317 (9317) & 99.99 & $9222(7654.8)$ & 99.74 \\
\hline soc-flixster & $3200(3200)$ & 10.87 & $3200 *(3200)$ & 10.68 & $3200 *(3200)$ & 10.99 & 3200 & 35.95 \\
\hline soc-lastfm & $1720 *(1720)$ & 4.82 & $1720 *(1720)$ & 6.03 & $1720 *(1720)$ & 6.03 & $1720(1720)$ & 15.41 \\
\hline soc-livejournal & $21935^{*}(\mathbf{2 1 9 3 5})$ & 42.96 & $21935^{*}(\mathbf{2 1 9 3 5})$ & 39.17 & $21935^{*}(\mathbf{2 1 9 3 5})$ & 38.35 & $21935 *(5755.6)$ & 89.72 \\
\hline soc-livejournal-user-groups & $877(828.7)$ & 76.43 & N/A (N/A) & N/A & N/A (N/A) & N/A & N/A (N/A) & N/A \\
\hline soc-ljournal-2008 & 39789 (39789) & 57.33 & $39789 *(39789)$ & 59.38 & $39789 *(39789)$ & 60.74 & $1448(948.222)$ & 97.51 \\
\hline soc-orkut & $4980(3559.8)$ & 91.36 & N/A (N/A) & N/A & N/A (N/A) & N/A & N/A (N/A) & N/A \\
\hline soc-orkut-dir & $4263(3596.22)$ & 88.26 & N/A (N/A) & N/A & N/A (N/A) & N/A & N/A (N/A) & N/A \\
\hline soc-pokec & $2925 *(2925)$ & 29.58 & $2925 *$ & 36.08 & $2925 *(2925)$ & 36.1 & $2003(1613.6)$ & 65.06 \\
\hline soc-sinaweibo & N/A (N/A) & N/A & N/A (N/A) & N/A & N/A (N/A) & N/A & N/A (N/A) & N/A \\
\hline soc-twitter-higgs & $7054 *(7054)$ & 12.56 & $7054 *(7054)$ & 18.27 & $7054 *(7054)$ & 18.09 & $3892(3865.3)$ & 55.38 \\
\hline soc-youtube & $1645(1645)$ & 2.87 & $1645 *(1645)$ & 2.38 & $1645 *(1645)$ & 2.79 & 1645 & 8.72 \\
\hline soc-youtube-snap & $1796(1796)$ & 4.25 & $1796 *(1796)$ & 4.59 & $1796 *(1796)$ & 4.79 & $1796(1796)$ & 12.73 \\
\hline socfb-A-anon & $2582 *(\mathbf{2 5 8 2})$ & 49.02 & $2582 *$ & 35.47 & $2582 *(\mathbf{2 5 8 2})$ & 34.58 & $2582(1847.2)$ & 81.47 \\
\hline socfb-B-anon & $2451 *(2451)$ & 24.43 & $2451 *$ & 32.51 & $2451 *$ & 31.28 & $2322(1722.4)$ & 78.65 \\
\hline socfb-uci-uni & $307(238.833)$ & 91.19 & N/A (N/A) & N/A & N/A (N/A) & N/A & N/A (N/A) & N/A \\
\hline tech-as-skitter & $6627 *(6627)$ & 7.32 & $6627 *$ & 22.69 & $6627 *(6627)$ & 22.79 & $6627(6627)$ & 76.24 \\
\hline tech-ip & $475(456.6)$ & 61.49 & N/A (N/A) & N/A & N/A (N/A) & N/A & $276(237.7)$ & 42.95 \\
\hline twitter_mpi & $4229(2922.9)$ & 90.29 & N/A (N/A) & N/A & N/A (N/A) & N/A & N/A (N/A) & N/A \\
\hline web-arabic-2005 & $10266 *(10266)$ & 0.11 & $10266 *(10266)$ & 1.42 & $10266 *(10266)$ & 1.49 & $10266 *(10266)$ & 2.17 \\
\hline web-baidu-baike & $2967 *(2967)$ & 22.02 & $2967 *$ & 25.89 & $2967 *(2967)$ & 25.59 & $1509(1434.3)$ & 66.08 \\
\hline web-it-2004 & $43259 *(43259)$ & 1.08 & $43259 *(43259)$ & 5.89 & $43259 *(43259)$ & 5.99 & $43259 *(43259)$ & 17.54 \\
\hline web-uk-2005 & $49947 *(49947)$ & 1.6 & $49947 *(49947)$ & 10.39 & $49947 *(49947)$ & 9.77 & $49947 *(49947)$ & 12.98 \\
\hline web-wikipedia-growth & $3107(3107)$ & 64.75 & $3107 *(3107)$ & 66.91 & $3107 *(3107)$ & 68.09 & 1675 & 98.06 \\
\hline web-wikipedia2009 & $3308 *(3308)$ & 8.02 & $3308 *$ & 7.79 & $3308 *(3308)$ & 7.49 & 3308 & 16.68 \\
\hline web-wikipedia_link & $\mathbf{8 5 8 0 7}(37968.8)$ & 78.38 & N/A (N/A) & N/A & 85694 (85694) & 99.58 & N/A (N/A) & N/A \\
\hline wikipedia_link_en & $\mathbf{4 3 8 9}$ (4386.5) & 75.8 & N/A (N/A) & N/A & $4389 *(4389)$ & 70.19 & 604 (453.7) & 91.14 \\
\hline
\end{tabular}




\subsubsection{RESULTS ON KONECT_NORMAL}

The results on the KONECT_normal benchmark are given in Table 6. Once again, the results of FastWClq are significantly better than those of the three competitors for most instances. FastWClq finds cliques of the same quality or better for all graphs except two: instance wikipedia_link_en that no algorithm is able to solve in the time limit, and instance flickr-links for which SCCWalk4L finds the better solution. FastWClq again computes the most number of optimal solutions, in this case 56 , although it only proves 44 of these solutions to be optimal. TSM-MWC and WLMC find fewer optimal solutions, at 48 each; however, all of these solutions are proved optimal. FastWClq also significantly outperforms the exact algorithms in terms of being able to produce any solution in the 100-second time limit: whereas FastWClq only fails to give a solution to one graph, TSM-MWC, WLMC fail on 29 instances each. SCCWalk4L fails to solve only 3 instances; however, its solution quality is worse than FastWClq on 38 instances.

Table 6. Experiment results of FastWClq and the state-of-the-art competitors on KONECT_normal benchmark.

\begin{tabular}{|c|c|c|c|c|c|c|c|c|}
\hline \multirow{2}{*}{ Instance } & \multicolumn{2}{|c|}{ FastWClq } & \multicolumn{2}{|c|}{ TSM-MWC } & \multicolumn{2}{|l|}{ WLMC } & \multicolumn{2}{|c|}{ SCCWalk4L } \\
\hline & size & time & size & time & size & time & size & time \\
\hline actor-collaboration & $29128 *(29128)$ & 9.1 & $29128 *(29128)$ & 27.39 & $29128 *(29128)$ & 27.69 & $29128(29128)$ & 80.24 \\
\hline amazon0601 & $1387 *(1387)$ & 1.54 & $1387 *(1387)$ & 2.88 & $1387 *(1387)$ & 3.09 & $1387 *(1387)$ & 4.07 \\
\hline as-skitter & $6540 *(6540)$ & 6.81 & $6540 *(6540)$ & 22.71 & $6540 *(6540)$ & 22.27 & $6540(6540)$ & 72.63 \\
\hline citeseer & $1292 *(\mathbf{1 2 9 2})$ & 1.32 & $1292 *(\mathbf{1 2 9 2})$ & 2.45 & $1292 *(\mathbf{1 2 9 2})$ & 2.46 & $1292(1267.1)$ & 20.87 \\
\hline com-amazon & $813 *(813)$ & 0.52 & $813 *(813)$ & 1.18 & $813^{*}(813)$ & 1.27 & $813^{*}(813)$ & 1.36 \\
\hline com-dblp & $11025 *(11025)$ & 0.26 & $11025 *(11025)$ & 1.27 & $11025 *(11025)$ & 1.35 & $11025^{*}(11025)$ & 1.64 \\
\hline com-youtube & $1672(1672)$ & 4.21 & $1672 *(1672)$ & 4.19 & $1672 *(1672)$ & 4.7 & $1672(1672)$ & 11.73 \\
\hline dbpedia-all & $1670 *(1670)$ & 16.29 & N/A (N/A) & N/A & N/A (N/A) & N/A & $1116(767.6)$ & 92.63 \\
\hline dbpedia-link & $784(563.667)$ & 95.24 & N/A (N/A) & N/A & N/A (N/A) & N/A & N/A (N/A) & N/A \\
\hline digg-friends & $3420(3420)$ & 25.4 & N/A (N/A) & N/A & N/A (N/A) & N/A & $323(233.5)$ & 9.64 \\
\hline douban & $1073 *(1073)$ & 0.06 & $1073 *(1073)$ & 0.52 & $1073 *(1073)$ & 0.68 & $1073(1073)$ & 0.61 \\
\hline elec & $1895 *(1895)$ & 0.15 & $1895 *(1895)$ & 2.68 & $1895^{*}(1895)$ & 2.79 & 1895 (1895) & 8.89 \\
\hline email-EuAll & $1637 *(1637)$ & 0.12 & $1637 *(1637)$ & 0.69 & $1637 *(1637)$ & 0.96 & 1637 (1637) & 0.98 \\
\hline enron & $3389 *(3389)$ & 0.14 & $3389 *(3389)$ & 18.55 & $3389 *(3389)$ & 18.64 & 3389 (3389) & 19.41 \\
\hline epinions & $10002(10002)$ & 0.24 & $10002 *(10002)$ & 0.96 & $10002 *(10002)$ & 1.05 & $10002(10002)$ & 4.88 \\
\hline facebook-wosn-links & $2993 *(2993)$ & 0.48 & $2993 *(2993)$ & 42.29 & $2993 *(2993)$ & 42.29 & 2993 (2993) & 27.45 \\
\hline facebook-wosn-wall & $1088 *(1088)$ & 0.55 & N/A (N/A) & N/A & N/A (N/A) & N/A & $428(313.7)$ & 24.55 \\
\hline flickr-growth & $10094(9970.9)$ & 37.88 & 8797 (8797) & 42.65 & 8797 (8797) & 40.93 & $8749(7097.2)$ & 98.49 \\
\hline flickr-links & 9760 (9577.7) & 26.2 & 8699 (8699) & 98.45 & $8272(8272)$ & 86.9 & $9822(8619)$ & 99.3 \\
\hline flickrEdges & $57102 *(57102)$ & 1.27 & $57102 *(57102)$ & 2.45 & $57102 *(57102)$ & 2.68 & $57102 *(57102)$ & 13.48 \\
\hline flixster & $3255(3255)$ & 10.48 & $3255^{*}(3255)$ & 10.18 & $3255^{*}(3255)$ & 10.84 & $3255(3255)$ & 23.51 \\
\hline hyves & $1798 *(1798)$ & 2.62 & $1798 *(1798)$ & 4.82 & $1798 *(1798)$ & 4.58 & 1798 (1798) & 6.68 \\
\hline lasagne-yahoo & $469 *(469)$ & 16.01 & $469 *(469)$ & 31.87 & $469 *(469)$ & 31.85 & $469(457.6)$ & 46.21 \\
\hline libimseti & 2028 (1910.3) & 56.71 & $2028 *(\mathbf{2 0 2 8})$ & 94.61 & $2028 *(\mathbf{2 0 2 8})$ & 94.34 & 2028 (2028) & 27.41 \\
\hline link-dynamic-dewiki & $9961(9748)$ & 75.16 & N/A (N/A) & N/A & N/A (N/A) & N/A & $285(239.714)$ & 94.32 \\
\hline link-dynamic-frwiki & $7832(7816)$ & 81.91 & N/A (N/A) & N/A & N/A (N/A) & N/A & $312(224.2)$ & 74.16 \\
\hline link-dynamic-itwiki & $4808(4808)$ & 28.04 & N/A (N/A) & N/A & N/A (N/A) & N/A & $438(275.7)$ & 42.23 \\
\hline link-dynamic-nlwiki & $5059 *(5059)$ & 15.42 & N/A (N/A) & N/A & N/A (N/A) & N/A & $234(202.7)$ & 29.23 \\
\hline link-dynamic-plwiki & $5183 *(5183)$ & 33.43 & N/A (N/A) & N/A & N/A (N/A) & N/A & $229(207.1)$ & 32.42 \\
\hline link-dynamic-simplewiki & $2697 *(2697)$ & $\mathbf{0 . 4 3}$ & $2697 *(2697)$ & 23.38 & $2697 *(2697)$ & 23.35 & $2697(2697)$ & 18.06 \\
\hline livejournal-links & $36124(36124)$ & 66.94 & $36124 *(36124)$ & 67.58 & $36124 *(36124)$ & 64.08 & $2150(1422.1)$ & 95.43 \\
\hline livemocha & $1736(1736)$ & 7.41 & $1736 *(1736)$ & 2.59 & $1736^{*}(1736)$ & 2.73 & $1736(1736)$ & 7.04 \\
\hline lkml-reply & $4992(4992)$ & 0.78 & N/A (N/A) & N/A & N/A (N/A) & N/A & $325(290.9)$ & 42.32 \\
\hline loc-gowalla_edges & $2767 *(\mathbf{2 7 6 7})$ & 0.33 & $2767 *(2767)$ & 1.26 & $2767 *(2767)$ & 1.46 & $2767(2662.8)$ & 39.42 \\
\hline munmun_digg_reply & $493 *(493)$ & 0.06 & $493 *(493)$ & 2.88 & $493 *(493)$ & 3 & $493(493)$ & 9.73 \\
\hline munmun_twitter_social & $617 *(617)$ & 0.41 & $617 *(617)$ & 1.19 & $617 *(617)$ & 1.29 & 617 (617) & 2.78 \\
\hline
\end{tabular}


Table 6. (Continued) Experiment results of FastWClq and the state-of-the-art competitors on KONECT_normal benchmark.

\begin{tabular}{|c|c|c|c|c|c|c|c|c|}
\hline \multirow{2}{*}{ Instance } & \multicolumn{2}{|l|}{ FastWClq } & \multicolumn{2}{|l|}{ TSM-MWC } & \multicolumn{2}{|l|}{ WLMC } & \multicolumn{2}{|l|}{ SCCWalk4L } \\
\hline & size & time & size & time & size & time & size & time \\
\hline orkut-links & $5246(4109.6)$ & 92.27 & N/A (N/A) & N/A & N/A (N/A) & N/A & N/A (N/A) & N/A \\
\hline patentcite & $1150 *(1150)$ & 28.84 & $1150 *(1150)$ & 28.28 & $1150 *(1150)$ & 29.39 & $1004(809.1)$ & 71.91 \\
\hline petster-carnivore & $115803 *(115803)$ & 11.49 & $115803 *(115803)$ & 53.78 & $115803 *(115803)$ & 53.88 & $6159(5547.7)$ & 99.38 \\
\hline petster-friendships-cat-uniq & 8453 (8415.9) & 22.36 & $8453 *(8453)$ & 45.78 & $8453 *(8453)$ & 44.79 & $2435(2325.8)$ & 95.58 \\
\hline petster-friendships-dog-uniq & $4622(4622)$ & 65.45 & $4622 *(4622)$ & 22.49 & $4622 *(4622)$ & 22.09 & $4622(4622)$ & 52.4 \\
\hline roadNet-CA & $503 *(503)$ & 1.52 & $503 *(503)$ & 4.19 & $503 *(503)$ & 4.29 & $503^{*}(503)$ & 6.23 \\
\hline roadNet-PA & $480 *(480)$ & 0.77 & $480 *(480)$ & 2.09 & $480 *(480)$ & 2.29 & $480 *(480)$ & 2.91 \\
\hline roadNet-TX & $484 *(484)$ & 0.89 & $484 *(484)$ & 2.59 & $484 *(484)$ & 2.69 & $484(484)$ & 3.59 \\
\hline slashdot-threads & $740 *(740)$ & 0.08 & $740 *(740)$ & 3.19 & $740 *(740)$ & 3.5 & $740(740)$ & 8.31 \\
\hline soc-LiveJournal1 & $32219(32219)$ & 56.21 & $32219 *(32219)$ & 58.65 & $32219 *(32219)$ & 57.59 & $4426(2005.4)$ & 94.61 \\
\hline soc-pokec-relationships & $2940 *(2940)$ & 24.95 & $2940 *(2940)$ & 33.69 & $2940 *(2940)$ & 34.49 & $2197(1664.8)$ & 66.32 \\
\hline trec-wt $10 \mathrm{~g}$ & $9009 *(9009)$ & 2.07 & $9009 *(9009)$ & 7.89 & $9009 *(9009)$ & 8.59 & 9009 (9009) & 16.11 \\
\hline web-BerkStan & 20098* (20098) & 1.31 & $20098 *(20098)$ & 25.68 & $20098 *(20098)$ & 25.49 & 20098 (20098) & 41.84 \\
\hline web-Google & $4110 *(4110)$ & 2.33 & $4110 *(4110)$ & 4.79 & $4110 *(4110)$ & 4.97 & $4110(4110)$ & 7.09 \\
\hline web-NotreDame & $15838 *(15838)$ & 0.17 & $15838 *(15838)$ & 1.29 & $15838 *(15$ & 1.39 & $15838 *(15838)$ & 2.65 \\
\hline web-Stanford & $6280 *(6280)$ & 0.48 & $6280 *(6280)$ & 4.59 & $6280 *(6280)$ & 4.67 & $6280(6280)$ & 20.59 \\
\hline wiki-Talk & 2714 (2714) & 16.98 & $2714 *(2714)$ & 14.79 & $2714 *(2714)$ & 14.29 & $2714(2714)$ & 23.78 \\
\hline wiki_talk_ar & 3338* (3338) & 2.12 & N/A (N/A) & N/A & N/A) & N/A & $336(229.5)$ & 10.65 \\
\hline wiki_talk_de & $5040(5040)$ & 8 & N/A (N/A) & N/A & /A) & N/A & 244 (214.9) & 8.5 \\
\hline wiki_talk_en & $4061(4055.8)$ & 68.7 & N/A (N/A) & N/A & N/A & N/A & $1494(1375.8)$ & 97.68 \\
\hline wiki_talk_es & 3675 (3675) & 4.21 & N/A (N/A) & N/A & N/A (N/A) & N/A & $388(249.4)$ & 6.57 \\
\hline wiki_talk_fr & 3879 (3879) & 5.76 & N/A (N/A) & N/A & N/A (N/A) & N/A & $262(211)$ & 7.2 \\
\hline wiki_talk_it & $4784(4784)$ & 3.81 & N/A (N/A) & N/A & N/A (N/A) & N/A & $288(224.5)$ & 13.71 \\
\hline wiki_talk_nl & 3891 (3891) & 2.62 & N/A (N/A) & N/A & N/A (N/A) & N/A & $341(253.6)$ & 6.62 \\
\hline wiki_talk_pt & $4498(4498)$ & 2.28 & N/A (N/A) & N/A & N/A (N/A) & N/A & $333(222.9)$ & 13.03 \\
\hline wiki_talk_ru & 3359 (3359) & 2.07 & N/A (N/A) & N/A & N/A (N/A) & N/A & $341(232.8)$ & 2.99 \\
\hline wiki_talk_zh & $3062(3062)$ & 2.42 & N/A (N/A) & N/A & N/A (N/A) & N/A & $333(220.7)$ & 6.93 \\
\hline wikipedia-growth & $3286(3286)$ & 46.35 & N/A (N/A) & $\mathrm{N}$ & /A) & N/A & $1638(1478.9)$ & 97.73 \\
\hline wikipedia_link_de & 83398* (83398) & 90.27 & N/A (N/A) & N/A & /A) & N/A & $1276(805.5)$ & 99.62 \\
\hline wikipedia_link_en & N/A (N/A) & N/A & N/A (N/A) & N/A & /A) & N/A & N/A (N/A) & N/A \\
\hline wikipedia_link_fr & $105526(40333.1)$ & 95.66 & N/A (N/A) & N/A & N/A (N/A) & N/A & N/A (N/A) & N/A \\
\hline wikipedia_link_it & $84676 *(84676)$ & 74.06 & N/A (N/A) & N/A & N/A (N/A) & N/A & $1710(862.333)$ & 99.1 \\
\hline wikipedia_link_ja & $84792 *(84792)$ & 76.13 & N/A (N/A) & N/A & N/A (N/A) & N/A & $2561(1687.5)$ & 98.5 \\
\hline wikipedia_link_pl & 82737 (82737) & 40.42 & N/A (N/A) & N/A & N/A (N/A) & N/A & 1985 (1585.9) & 94.31 \\
\hline wikipedia_link_pt & $104461 *(104461)$ & 40.61 & N/A (N/A) & N/A & N/A (N/A) & N/A & $13939(3933.1)$ & 88.49 \\
\hline wikipedia_link_ru & 80185 (80185) & 73.62 & N/A (N/A) & N/A & N/A (N/A) & N/A & $1771(872.125)$ & 98.65 \\
\hline wikisigned-k2 & $1192(1192)$ & 1.01 & $1192 *(1192)$ & 3.99 & $1192 *(1192)$ & 4.09 & $1192(1192)$ & 17.92 \\
\hline wordnet-words & $3274 *(3274)$ & 0.2 & $3274 *(3274)$ & 0.69 & $3274 *(3274)$ & 0.79 & $3274 *(3274)$ & 1.09 \\
\hline youtube-links & $1648(1648)$ & 2.86 & $1648 *(1648)$ & 4.58 & $1648 *(1648)$ & 4.7 & & 12.43 \\
\hline youtube-u-growth & $2015(2015)$ & 11.06 & $2015 *(2015)$ & 28.79 & $2015 *(2015)$ & 28.19 & 1999 (1999) & 51.05 \\
\hline zhishi-baidu-internallink & $3166 *(3166)$ & 21.65 & $3166 *(3166)$ & 49.58 & $3166^{*}(3166)$ & 47.39 & $1476(1464)$ & 66.6 \\
\hline zhishi-baidu-relatedpages & $9154 *(\mathbf{9 1 5 4})$ & 1.33 & $9154 *(\mathbf{9 1 5 4})$ & 49.89 & $9154 *(\mathbf{9 1 5 4})$ & 49.59 & $9154(5904.3)$ & 65.04 \\
\hline zhishi-hudong-internallink & $26627 *(\mathbf{2 6 6 2 7})$ & 21.2 & $26627 *(26627)$ & 33.19 & $26627 *(\mathbf{2 6 6 2 7})$ & 31.79 & $26627 *(24272.3)$ & 82.93 \\
\hline zhishi-hudong-relatedpages & $1566 *(\mathbf{1 5 6 6})$ & 29.66 & $1566 *(\mathbf{1 5 6 6})$ & 48.49 & $1566^{*}(\mathbf{1 5 6 6})$ & 50.98 & $1566(1121.5)$ & 70.15 \\
\hline
\end{tabular}

\subsection{The Effectiveness of Three Upper Bound Functions}

To determine the effectiveness of three upper bound functions (i.e., $U B_{0}, U B_{1}$ and $U B_{2}$ ), we compare FastWClq with its five alternative versions, each of which uses a different subset of the upper bound functions, given as follows: (1) FastWClq0 (only $U B_{0}$ ); (2) FastWClq1 (only $U B_{1}$ ); (3) FastWClq2 (only $U B_{2}$ ); (4) FastWClq01 (only $U B_{0}$ and $U B_{1}$ ); and (5) FastWClq02 (only $U B_{0}$ and 
Table 7. Comparative results of solving ability of FastWClq and its variants with each upper bound and each combination of two upper bounds on all benchmarks

\begin{tabular}{|c|c|c|c|c|c|c|c|c|c|c|c|c|c|}
\hline \multirow{2}{*}{ Dellc } & \multirow{2}{*}{ nst. } & \multicolumn{2}{|c|}{ FastWClq } & \multicolumn{2}{|c|}{ FastWClq02 } & \multicolumn{2}{|c|}{ FastWClq01 } & \multicolumn{2}{|c|}{ FastWClq2 } & \multicolumn{2}{|c|}{ FastWClq1 } & \multicolumn{2}{|c|}{ FastWClq0 } \\
\hline & & $\# \mathrm{u}$ & & $\# \mathrm{u}$ & time & $11 \mathrm{wn}$ & me & \#wi & time & \#wi & ime & \#wi & บाก \\
\hline & & 47 & 2.53 & 46 & 32.97 & 47 & 33.51 & 48 & 29.9 & 49 & 29.72 & 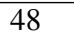 & 2.25 \\
\hline & 6 & & & & & & & & 2 & & 29.42 & & . \\
\hline & 80 & & & 1 & & & & & 20.93 & & 20.65 & & 2.62 \\
\hline KONECT_normal & 80 & 70 & 21.27 & 70 & 21.84 & 69 & 21.63 & 51 & 20.59 & 58 & 20.39 & 70 & 21.34 \\
\hline
\end{tabular}

Table 8. Comparative results of proving ability of FastWClq and its variants with each upper bound and each combination of two upper bounds on all benchmarks

\begin{tabular}{llllllll}
\hline Benchmark & \#inst. & FastWClq FastWClq02 & FastWClq01 & FastWClq2 & FastWClq1 FastWClq0 \\
\cline { 3 - 7 } & & \#prov & \#prov & \#prov & \#prov & \#prov & \#prov \\
\hline Repository_200 & 65 & 36 & 36 & 22 & 35 & 22 & 12 \\
Repository_normal & 65 & 36 & 36 & 19 & 36 & 19 & 14 \\
KONECT_200 & 80 & 42 & 41 & 17 & 34 & 14 & 16 \\
KONECT_normal & 80 & 44 & 44 & 14 & 38 & 11 & 13 \\
\hline
\end{tabular}

$U B_{2}$ ). For each algorithm, we report the number of instances where it performs best among all algorithms on the metric of maximum and average weight of cliques, denoted by '\#win', and the number of instances where the algorithm proves the optimality of its solution, denoted as '\#prov'.

Table 7 summarizes the comparative results in terms of solution quality and run time for FastWClq and the five alternative versions on all four benchmarks. Overall, FastWClq gives the best performance on these benchmarks, thus showing that all upper bounds contribute to its performance.

Although FastWClq is slightly outperformed in terms of quality by other variations on some individual benchmarks, FastWClq outperforms these other variants on all benchmarks in terms of proving optimality. In Table 8 we present the number of solutions for which FastWClq and its five versions prove optimality. Observed from Table 8, FastWClq shows the best proving ability, while the version using $U B_{0}$ and $U B_{2}$ has very close proving ability with FastWClq. This indicates that with $U B_{0}$ and $U B_{2}$, the contribution of $U B_{1}$ on the proving ability can be omitted. This can be well understood as $U B_{2}$ is an enhanced bound built on the basis of $U B_{1}$.

To further analyze the upper bound functions of FastWClq, we report the percent of remaining vertices after the last successful call of graph reduction and the time at which the last successful reduction procedure finishes (Table 9). The detailed information of all instances is presented in Figure 2. Observed from the results, the upper bound functions can make a significant contribution for FastWClq in a short time.

\subsection{Summary of Experiments}

Table 10 summarizes the experimental results of FastWClq and three competitors on the four benchmarks. To further provide a better understanding of the performance of the algorithms, besides the time limit of 100 seconds, we also test the algorithms with the time limit of 300 seconds and 600 seconds. For these four benchmarks of large graphs, FastWClq has the top performance in terms of solution quality, followed by TSM-MWC, WLMC and SCCWalk4L (in this order). 
Table 9. Information on the effectiveness of graph reduction in FastWClq. ( $R V_{\text {avg }}$ is the average number of remaining vertices, $R V_{\text {avg }} \%$ is the percent of remaining vertices among all the vertices, and $t_{\text {avg }}$ is the time at which the last successful reduction procedure finishes.)

\begin{tabular}{llll}
\hline Benchmark & \multicolumn{3}{l}{ FastWClq } \\
\cline { 2 - 4 } & $R V_{\text {avg }}$ & $R V_{\text {avg }} \%$ & $t_{\text {avg }}$ \\
\hline Repository_200 & 2025185.44 & $10.52 \%$ & 40.12 \\
Repository_normal & 2031723.03 & $11 \%$ & 41.13 \\
KONECT_200 & 413092.01 & $4.65 \%$ & 29.68 \\
KONECT_normal & 412874.82 & $4.58 \%$ & 29.19 \\
\hline
\end{tabular}

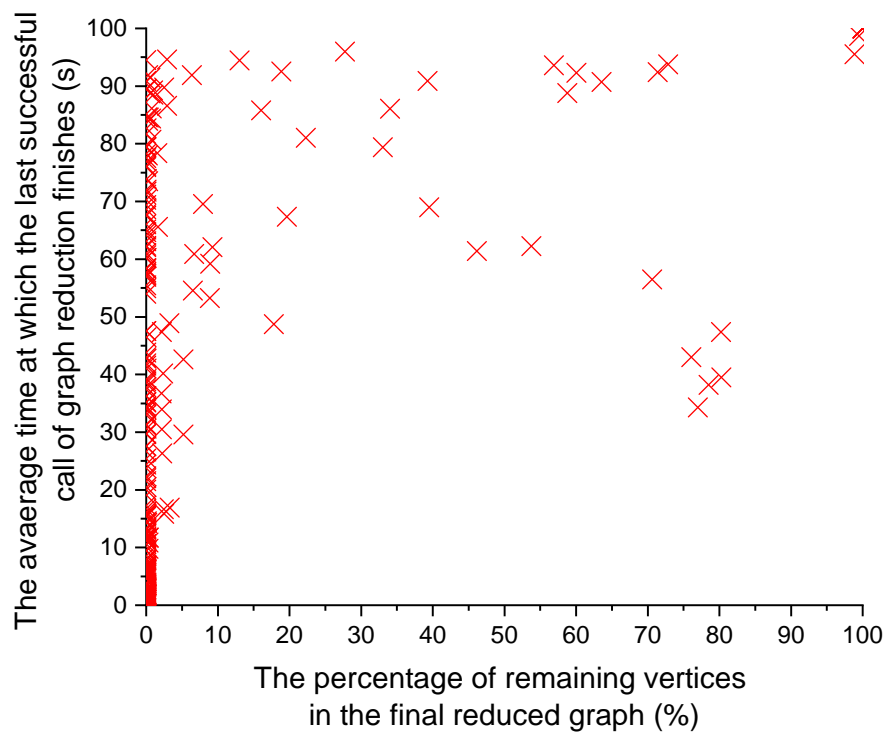

Figure 2. A plot comparing the time of the last successful graph reduction and the percentage of vertices remaining in the graph.

The density of these large real-world graphs ranges from $0.000004 \%$ to $0.139 \%$. For 75 large real-world graphs whose density is less than $0.001 \%$, FastWClq steadily obtains the same or better solution values, compared to all competitors. For the remaining graphs whose density, FastWClq is also competitive, and gives the best solution for those instances with only 7 exceptions.

We now turn to compare the ablity of proving the optimality. Compared with FastWClq, exact algorithms TSM-MWC and WLMC prove the optimality of more solutions, which is not surprising. Nevertheless, we note that our semi-exact algorithm FastWClq also shows competitive proving ability. It proves the optimality of its solutions for about half of the tested graphs including graphs with millions of vertices, and the average time for doing this is less than 22 seconds.

\section{Conclusions and Future Work}

This paper presented a novel semi-exact method for the maximum weight clique problem (MWCP), which aims to solve large graphs within a short time limit. The method interleaves clique finding 
Table 10. Experimental results of FastWClq and the competitors on all benchmarks with different time limits.

\begin{tabular}{|c|c|c|c|c|c|c|c|c|c|c|c|c|c|}
\hline \multirow[t]{2}{*}{ Benchmark } & \multirow[t]{2}{*}{ \#inst. } & \multirow{2}{*}{$\begin{array}{l}\text { Time } \\
\text { Limit }\end{array}$} & \multicolumn{3}{|c|}{ FastWClq } & \multicolumn{3}{|c|}{ TSM-MWC } & \multicolumn{3}{|c|}{ WLMC } & \multicolumn{2}{|c|}{ SCCWalk4L } \\
\hline & & & \#win & time & \#prov & \#win & time & \#prov & \#win & time & \#prov & \#win & time \\
\hline Repository_200 & 65 & \multirow{4}{*}{$100 \mathrm{~s}$} & 55 & 32.53 & 36 & 45 & 26.19 & 45 & 53 & 31.32 & 52 & 22 & 54.7 \\
\hline Repository_normal & 65 & & 54 & 32.34 & 36 & 45 & 25.28 & 45 & 52 & 31.6 & 52 & 23 & 54.81 \\
\hline KONECT_200 & 80 & & 75 & 22.34 & 42 & 48 & 22 & 48 & 47 & 23.09 & 47 & 37 & 39.34 \\
\hline KONECT_normal & 80 & & 75 & 21.27 & 44 & 48 & 20.9 & 48 & 48 & 20.58 & 48 & 34 & 39.67 \\
\hline Repository_200 & 65 & \multirow{4}{*}{$300 \mathrm{~s}$} & 55 & 50.26 & 43 & 52 & 46.66 & 51 & 62 & 53.38 & 62 & 37 & 121.37 \\
\hline Repository_normal & 65 & & 54 & 45.35 & 45 & 51 & 43.58 & 51 & 62 & 48.77 & 62 & 33 & 131.74 \\
\hline KONECT_200 & 80 & & 76 & 31.86 & 52 & 59 & 61.8 & 58 & 58 & 62.19 & 58 & 47 & 86.47 \\
\hline KONECT_normal & 80 & & 76 & 30.31 & 52 & 61 & 58.6 & 61 & 59 & 57.83 & 59 & 50 & 89.68 \\
\hline Repository_200 & 65 & \multirow{4}{*}{$600 \mathrm{~s}$} & 59 & 67.88 & 43 & 52 & 46.66 & 51 & 62 & 55.49 & 62 & 38 & 182.67 \\
\hline Repository_normal & 65 & & 58 & 81.34 & 45 & 52 & 49.29 & 52 & 62 & 48.77 & 62 & 36 & 210.17 \\
\hline KONECT_200 & 80 & & 77 & 39.52 & 52 & 62 & 70 & 62 & 61 & 75.23 & 61 & 57 & 140.92 \\
\hline KONECT_normal & 80 & & 77 & 50 & 52 & 62 & 63.07 & 62 & 61 & 80.43 & 61 & 55 & 140.7 \\
\hline
\end{tabular}

and graph reduction. Several ideas were proposed to improve the clique finding algorithm and the graph reduction algorithm. The resulting algorithm is called FastWClq. Experiments on large realworld graphs show that FastWClq finds better solutions than state-of-the-art algorithms while using less time on most instances. Also, FastWClq proves the optimality of its solutions for about half of the tested graphs including graphs with millions of vertices, and the average time for doing this is less than 22 seconds.

As shown in this work, semi-exact algorithms take the advantage of solving and proving, and seem a promising direction for solving large combinatorial optimization problems. A significant direction for future work is to apply the semi-exact method to other combinatorial optimization problems problems.

\section{Acknowledgement}

This work is an improved and extended version of a conference paper (Cai \& Lin, 2016). This work was supported by Beijing Academy of Artificial Intelligence (BAAI), Youth Innovation Promotion Association, Chinese Academy of Sciences [No. 2017150], NSFC Grant 61806050, and Jilin Science and Technology Association QT202005.

\section{References}

Balasundaram, B., \& Butenko, S. (2006). Graph domination, coloring and cliques in telecommunications. In Handbook of Optimization in Telecommunications, pp. 865-890.

Ballard, D., \& Brown, C. (1982). Computer Vision. New Jersey: Prentice Hall.

Batagelj, V., \& Zaveršnik, M. (2003). An $O(m)$ algorithm for cores decomposition of networks. CoRR, cs.DS/0310049.

Benlic, U., \& Hao, J.-K. (2013). Breakout local search for maximum clique problems. Computers \& Operations Research, 40(1), 192-206.

Busygin, S. (2006). A new trust region technique for the maximum weight clique problem. Discrete Applied Mathematics, 154(15), 2080-2096. 
Cai, S. (2015). Balance between complexity and quality: Local search for minimum vertex cover in massive graphs. In Proceedings of IJCAI 2015, pp. 747-753.

Cai, S., Li, Y., Hou, W., \& Wang, H. (2019). Towards faster local search for minimum weight vertex cover on massive graphs. Information Sciences, 471, 64-79.

Cai, S., \& Lin, J. (2016). Fast solving maximum weight clique problem in massive graphs. In Proceedings of IJCAI 2016, pp. 568-574.

Cai, S., Luo, C., Zhang, X., \& Zhang, J. (2021). Improving local search for structured sat formulas via unit propagation based construct and cut initialization. In Proceedings of CP 2021.

Demange, M., de Werra, D., Monnot, J., \& Paschos, V. T. (2002). Weighted node coloring: when stable sets are expensive. In International Workshop on Graph-Theoretic Concepts in Computer Science, pp. 114-125. Springer.

Fan, Y., Li, N., Li, C., Ma, Z., Latecki, L. J., \& Su, K. (2017). Restart and random walk in local search for maximum vertex weight cliques with evaluations in clustering aggregation. In Proceedings of IJCAI 2017, pp. 622-630.

Fang, Z., Li, C.-M., Qiao, K., Feng, X., \& Xu, K. (2014). Solving maximum weight clique using maximum satisfiability reasoning. In Proceedings of ECAI 2014, pp. 303-308.

Fellows, M. R., \& Downey, R. (1998). Parameterized Complexity. Springer.

Gomez Ravetti, M., \& Moscato, P. (2008). Identification of a 5-protein biomarker molecular signature for predicting Alzheimer's disease. PloS one, 3(9), e3111.

Guturu, P., \& Dantu, R. (2008). An impatient evolutionary algorithm with probabilistic tabu search for unified solution of some NP-hard problems in graph and set theory via clique finding. IEEE Trans. Systems, Man, and Cybernetics, Part B, 38(3), 645-666.

Hsu, H., \& Chang, G. J. (2016). Max-coloring of vertex-weighted graphs. Graphs and Combinatorics, 32(1), 191-198.

Jiang, H., Li, C., Liu, Y., \& Manyà, F. (2018). A two-stage MaxSAT reasoning approach for the maximum weight clique problem. In Proceedings of AAAI 2018, pp. 1338-1346.

Jiang, H., Li, C., \& Manyà, F. (2017). An exact algorithm for the maximum weight clique problem in large graphs. In Proceedings of AAAI 2017, pp. 830-838.

Karp, R. M. (1972). Reducibility among combinatorial problems. In Complexity of computer computations, pp. 85-103. Springer.

Konc, J., \& Janezic, D. (2007). An improved branch and bound algorithm for the maximum clique problem. Communications in Mathematical and in Computer Chemistry, 58, 569-590.

Kumlander, D. (2004). Fast maximum clique algorithms for large graphs. In Proceedings of the fourth conference on engineering computational technology, pp. 202-208.

Kunegis, J. (2013). Konect: the koblenz network collection. In Proceedings of $W W W 2013$, pp. $1343-1350$.

Lamm, S., Schulz, C., Strash, D., Williger, R., \& Zhang, H. (2019). Exactly solving the maximum weight independent set problem on large real-world graphs. In Proceedings of ALENEX 2019, pp. 144-158. 
Li, C.-M., Fang, Z., \& Xu, K. (2013). Combining MaxSAT reasoning and incremental upper bound for the maximum clique problem. In Proceedings of ICTAI 2013, pp. 939-946.

Li, C.-M., Jiang, H., \& Manyà, F. (2017). On minimization of the number of branches in branchand-bound algorithms for the maximum clique problem. Computers \& Operations Research, $84,1-15$.

Li, C., Liu, Y., Jiang, H., Manyà, F., \& Li, Y. (2018). A new upper bound for the maximum weight clique problem. European Journal of Operational Research, 270(1), 66-77.

Li, C. M., \& Quan, Z. (2010). An efficient branch-and-bound algorithm based on MaxSAT for the maximum clique problem. In Proceedings of AAAI 2010, pp. 128-133.

Li, R., Hu, S., Cai, S., Gao, J., Wang, Y., \& Yin, M. (2020). Numwvc: A novel local search for minimum weighted vertex cover problem. Journal of the Operational Research Society, 71(9), 1498-1509.

Massaro, A., Pelillo, M., \& Bomze, I. M. (2002). A complementary pivoting approach to the maximum weight clique problem. SIAM Journal on Optimization, 12(4), 928-948.

McCreesh, C., Prosser, P., Simpson, K., \& Trimble, J. (2017). On maximum weight clique algorithms, and how they are evaluated. In Proceedings of CP 2017, pp. 206-225.

Östergård, P. R. J. (1999). A new algorithm for the maximum-weight clique problem. Electronic Notes in Discrete Mathematics, 3, 153-156.

Pullan, W. (2006). Phased local search for the maximum clique problem. Journal of Combinatorial Optimization, 12(3), 303-323.

Pullan, W. (2008). Approximating the maximum vertex/edge weighted clique using local search. Journal of Heuristics, 14(2), 117-134.

Pullan, W. (2009). Optimisation of unweighted/weighted maximum independent sets and minimum vertex covers. Discrete Optimization, 6(2), 214-219.

Pullan, W., \& Hoos, H. H. (2006). Dynamic local search for the maximum clique problem. Journal of Artificial Intelligence Research, 25, 159-185.

Rossi, R. A., \& Ahmed, N. K. (2015). The network data repository with interactive graph analytics and visualization. In Proceedings of AAAI 2015, pp. 4292-4293.

Rossi, R. A., Gleich, D. F., Gebremedhin, A. H., \& Patwary, M. (2014). Fast maximum clique algorithms for large graphs. In Proceedings of WWW 2014, pp. 365-366.

San Segundo, P., Furini, F., \& Artieda, J. (2019). A new branch-and-bound algorithm for the maximum weighted clique problem. Computers \& Operations Research, 110, 18-33.

San Segundo, P., Lopez, A., \& Pardalos, P. M. (2016). A new exact maximum clique algorithm for large and massive sparse graphs. Computers \& Operations Research, 66, 81-94.

San Segundo, P., Matía, F., Rodríguez-Losada, D., \& Hernando, M. (2013). An improved bit parallel exact maximum clique algorithm. Optimization Letters, 7(3), 467-479.

Seidman, S. (1983). Network structure and minimum degree. Social Networks, 5(3), 269-287.

Singh, A., \& Gupta, A. K. (2006a). A hybrid evolutionary approach to maximum weight clique problem. International Journal of Computational Intelligence Research, 2(4), 349-355. 
Singh, A., \& Gupta, A. K. (2006b). A hybrid heuristic for the maximum clique problem. Journal of Heuristics, 12(1-2), 5-22.

Tomita, E., \& Kameda, T. (2007). An efficient branch-and-bound algorithm for finding a maximum clique with computational experiments. Journal of Global optimization, 37(1), 95-111.

Tomita, E., \& Seki, T. (2003). An efficient branch-and-bound algorithm for finding a maximum clique. In Discrete mathematics and theoretical computer science, pp. 278-289.

Tomita, E., Sutani, Y., Higashi, T., Takahashi, S., \& Wakatsuki, M. (2010). A simple and faster branch-and-bound algorithm for finding a maximum clique. In Proceedings of WALCOM 2010, pp. 191-203.

Verma, A., Buchanan, A., \& Butenko, S. (2015). Solving the maximum clique and vertex coloring problems on very large sparse networks. INFORMS Journal on Computing, 27(1), 164-177.

Wang, Y., Cai, S., Chen, J., \& Yin, M. (2020). SCCWalk: An efficient local search algorithm and its improvements for maximum weight clique problem. Artificial Intelligence, 280, 103230.

Wang, Y., Cai, S., \& Yin, M. (2016). Two efficient local search algorithms for maximum weight clique problem. In Proceedings of AAAI 2016, pp. 805-811.

Wu, Q., Hao, J.-K., \& Glover, F. (2012). Multi-neighborhood tabu search for the maximum weight clique problem. Annals of Operations Research, 196(1), 611-634.

Zhou, Y., Hao, J., \& Goëffon, A. (2017). PUSH: A generalized operator for the maximum vertex weight clique problem. European Journal of Operational Research, 257(1), 41-54.

Zuckerman, D. (2007). Linear degree extractors and the inapproximability of max clique and chromatic number. Theory of Computing, 3(1), 103-128. 